\title{
Development of a model describing regulation of casein synthesis by the mammalian target of rapamycin (mTOR) signaling pathway in response to insulin, amino acids, and acetate
}

\author{
J. J. Castro, ${ }^{1}$ S. I. Arriola Apelo, ${ }^{2}$ J. A. D. R. N. Appuhamy, and M. D. Hanigan \\ Department of Dairy Science, Virginia Tech, Blacksburg 24061
}

\begin{abstract}
To improve dietary protein use efficiency in lactating cows, mammary protein synthesis responses to AA, energy substrates, and hormones must be better understood. These entities exert their effects through stimulation of mRNA translation via control of initiation and elongation rates at the cellular level. A central protein kinase of this phenomenon is the mammalian target of rapamycin (mTOR), which transfers the nutritional and hormonal stimuli onto a series of proteins downstream through a cascade of phosphorylation reactions that ultimately affect protein synthesis. The objective of this work was to further develop an existing mechanistic model of mTOR phosphorylation responses to insulin and total essential AA to include the effects of specific essential AA and acetate mediated by signaling proteins including protein kinase B (Akt), adenosine monophosphate activated protein kinase (AMPK), and mTOR and to add a representation of milk protein synthesis. Data from 6 experiments in MAC-T cells and mammary tissue slices previously conducted in our laboratory were assembled and used to parameterize the dynamic system of differential equations representing Akt, AMPK, and mTOR in their phosphorylated and dephosphorylated states and the resulting regulation of milk protein synthesis. The model predicted phosphorylated Akt, mTOR, AMPK, and casein synthesis rates with root mean square prediction errors of 16.8, $28.4,33.0$, and $54.9 \%$, respectively. All other dependent variables were free of mean and slope bias, indicating an adequate representation of the data. Whereas mTOR was not very sensitive to changes in insulin or acetate levels, it was highly sensitive to leucine and isoleucine, and this signal appeared to be effectively transduced to casein synthesis. Although prior work had observed
\end{abstract}

\footnotetext{
Received November 2, 2015.

Accepted April 19, 2016.

${ }^{1}$ Corresponding author: cjuan3@vt.edu

${ }^{2}$ Current address: Madison School of Medicine and Public Health, University of Wisconsin, Madison 53711.
}

a relationship with additional essential $\mathrm{AA}$, and data supporting those conclusions were present in the data set, we were unable to derive significant relationships with any essential AA other than leucine and isoleucine. The signaling properties and dynamics of AMPK under nutrient depletion and sufficiency, the responses to additional essential AA, and the consequent effects on protein synthesis remain to be better understood.

Key words: amino acids, mTOR, casein, insulin

\section{INTRODUCTION}

Dairy cattle convert dietary nitrogen to milk nitrogen at an efficiency of approximately $25 \%$ under current feeding conditions (Kebreab et al., 2001; Arriola Apelo et al., 2014a). Half of this loss is due to clearance of AA from blood by liver and gut tissues (Hanigan et al., 2004), and most of it takes place after first pass through those tissues (MacRae et al., 1997; Hanigan et al., 2004). If mammary responses to specific EAA could be more precisely predicted, dietary protein and AA could be reduced, thus leading to lower AA catabolism by gut and liver. Understanding mammary protein synthesis responses to individual AA and its modulation by energy substrates and hormones may help us achieve this goal and in turn allow us to optimally allocate certain nutrients to achieve better efficiency while minimizing production costs.

Mammalian target of rapamycin (mTOR) modulates mRNA translation via control of initiation and elongation rates (Wang and Proud, 2006) in mammary cellular systems (Moshel et al., 2006). Its activity is controlled by phosphorylation in response to several stimuli. Effectors of mTOR phosphorylation include insulin, cellular energy status, and multiple EAA (Appuhamy et al., 2012), with branched-chain AA (BCAA) responses being more documented (Kimball and Jefferson, 2006). Insulin acts via the protein kinase B (Akt) pathway to stimulate mTOR phosphorylation (Suryawan et al., 2009) when cellular energy supply is adequate. Conversely, the AMP-activated kinase (AMPK) pathway becomes active, phosphorylated, when energy concen- 
trations are low (e.g., increased ADP/ATP) and inhibits mTOR phosphorylation, thus downregulating the protein synthetic machinery (Wang and Proud, 2006; Viollet et al., 2010). Conversely, abundance of glucose for example, reduces AMPK activation in tissues such as muscle, liver, and kidney, thereby releasing the inhibition of mTOR phosphorylation (Viollet et al., 2010). Amino acids act through mTOR phosphorylation to inhibit the eukaryotic initiation factor $4 \mathrm{E}$ binding protein-1 (4eBP1), thus enhancing protein synthesis (Kimball and Jefferson, 2006). Other downstream mediators of mTOR include protein S6 kinase 1 (S6k1), which phosphorylates ribosomal protein $\mathrm{S} 6(\mathbf{r p S 6})$ and eukaryotic elongation factor 2 (eEF2; Arriola Apelo et al., 2014a). Leucine feeding in neonates can increase muscle protein synthesis by stimulating translation initiation and, perhaps, also reducing protein degradation (Boutry et al., 2013).

We previously conducted a series of experiments in MAC-T cells and lactogenic mammary tissue slices to explore the responsiveness of the mTOR signaling pathway and casein synthesis to individual EAA, insulin, acetate, and glucose (Appuhamy et al., 2011, 2012, 2014; Appuhamy and Hanigan, 2011; Arriola Apelo et al., 2014b,c). In general, mTOR phosphorylation responses to these stimuli were saturable and independent (i.e., the effect of one nutrient does not depend on the level of another) with exceptions. Whereas our experimental observations on the effect of EAA, energy substrates, and insulin on protein synthesis regulation qualitatively align with reports in the literature, it could be useful to integrate such data to test quantitative relationships between nutrients, mTOR signaling, and protein synthesis and assess the sensitivity of the latter to nutrient inputs in order to identify what areas remain incompletely understood.

We previously constructed a mathematical model of mTOR phosphorylation responses to insulin and total EAA (Appuhamy and Hanigan, 2011). The objective of this work was to extend that model to include the effects of specific EAA and energy substrates on mTOR phosphorylation, and link mTOR signaling with casein synthesis rates. Mediator signaling proteins downstream of mTOR that were evaluated included 4eBP1, S6k1, eEF2, and rpS6.

\section{MATERIALS AND METHODS}

\section{Model Description}

The initial full model, as depicted in Figure 1, represented our initial hypotheses and was conceptualized from the experimental observations collected previously in our laboratory. The model represents the Akt- and
AMPK-mediated regulatory effect of insulin as well as glucose and acetate, respectively, and the direct effects of Leu, Ile, Thr, Met, and other EAA on mTOR phosphorylation and its subsequent modulation of casein synthesis rates through signaling by $4 \mathrm{eBP} 1, \mathrm{~S} 6 \mathrm{k} 1, \mathrm{eEF} 2$, and rpS6. Indeed, Appuhamy et al. (2012) found that Ile, Leu, Thr, Met, Arg, and Trp or a combination of all EAA significantly affected mTOR phosphorylation in MAC-T cells or mammary tissue slices (MTS), and casein synthesis in MTS; Appuhamy et al. (2011) found that both an EAA mixture and insulin significantly affected phosphorylation of the mTOR pathway in both MAC-T and MTS, and casein synthesis in MTS; and Appuhamy et al. (2014) observed that acetate, EAA, or glucose numerically reduced AMPK phosphorylation by about $16 \%$ in MTS, but casein synthesis was unaffected. These relationships were tested in the present work by fitting the model to the observed experimental data. The model was then reduced to keep only those aspects that were statistically significant, or those nonsignificant but of biological relevance for sensitivity analysis and discussion of remaining knowledge gaps.

This full model was a dynamic system of 14 differential equations coded in ACSLX (Version 3.1.4.2, Aegis Technologies Group, Huntsville, AL) corresponding to 14 state variables representing each of Akt, AMPK, mTOR, 4eBP1, eEF2, S6k1, and rpS6 proteins in their phosphorylated and dephosphorylated states. The time units were hours and the Runge-Kutta-Fehlberg variable step size algorithm was used to perform the numerical integration with a maximum step size of $0.001 \mathrm{~h}$. A reference state model was established using extracellular concentrations of insulin, Ile, Leu, and acetate at $10 \mu \mathrm{g} / \mathrm{dL}, 212 \mu \mathrm{M}, 270 \mu \mathrm{M}$, and $2.5 \mathrm{mM}$, respectively.

The differential equations describing the change of the phosphorylated and dephosphorylated forms of each protein with respect to time were based on the assumption that the bidirectional fluxes for each protein were equal under the reference steady state conditions following the same general principle from Appuhamy and Hanigan (2011). These bidirectional fluxes were used to define the differential equations for the phosphorylated form of each protein:

$$
\frac{\mathrm{d} Q_{\text {Protein }-P}}{\mathrm{~d} t}=F_{\text {Protein-UP }}-F_{\text {Protein }-P U},
$$

where $Q_{\text {PROTEIN-P }}$ refers to the pool size of each phosphorylated $(P)$ protein with Protein representing Akt, AMPK, mTOR, 4eBP1, eEF2, S6k1, and rpS6; $F_{\text {Protein }}$ represented the flux between the phosphorylated and dephosphorylated protein pools, with the subscripts $P$ 


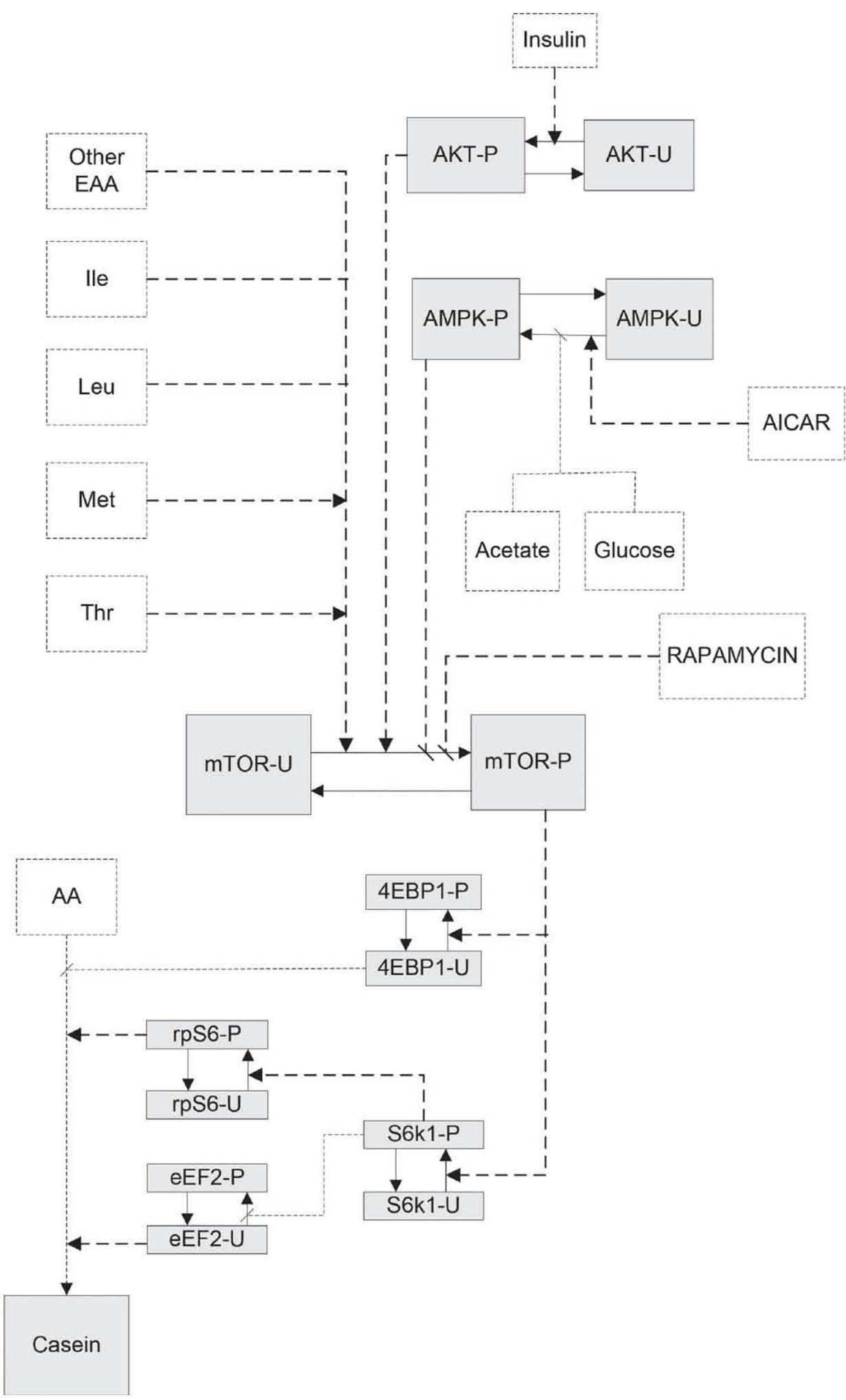

Figure 1. A schematic of the full model based on previous experimental knowledge. Boxes enclosed by dashed lines are model inputs; boxes enclosed by solid lines are state variables; solid arrows denote fluxes; dashed arrows denote regulation with an arrow line endpoint indicating a positive regulator and an angled bar endpoint denoting an inhibitory regulator. Akt = protein kinase B; AMPK = AMP-activated protein kinase; $\mathrm{mTOR}=$ mammalian target of rapamycin; 4eBP1 = eukaryotic translation initiation factor binding protein; S6k1 = ribosomal protein $\mathrm{S} 6$ kinase; rpS6 = ribosomal protein S6; eEF2 = eukaryotic elongation factor. Phoshorylated signaling proteins are denoted by a terminal $\mathrm{P}$ in the name and unphosphorylated forms by a terminal $\mathrm{U}$ in the name. 
and $U$ denoting each pool, respectively. Thus, $F_{\text {Protein }}{ }^{-}$ $U P$ and $F_{\text {Protein-PU }}$ refer to the flux from the unphosphorylated to the phosphorylated form, and vice versa, respectively.

It was assumed that the total protein pool size (i.e., phosphorylated plus unphosphorylated form of a given protein) did not change during the course of the 4-h experiments, and thus the unphosphorylated protein differential was equal to the negative value of the phosphorylated differential:

$$
\frac{\mathrm{d} Q_{\text {Protein }-U}}{\mathrm{~d} t}=\frac{-\mathrm{d} Q_{\text {Protein }-P}}{\mathrm{~d} t}
$$

The kinase flux equations were constructed using both mass action,

$$
F_{\text {Protein- } U P}=Q_{\text {Protein- } P} \times k_{\text {Protein- } U P}+\text { Intercept },
$$

and Michaelis-Menten (M-M) forms,

$$
F_{\text {PROTEIN-UP }}=\left[\frac{V_{M a x-U P}}{1+\sum_{i=1}^{n} \frac{K_{m_{n}}}{C_{n}}}\right]+\text { Intercept }
$$

where $k_{\text {Protein }^{-} U P}$ and Intercept are the rate constant for the phosphorylation reaction and the basal phosphorylation rate in equation [3], respectively, and $V_{M a x-U P}$, $K_{m_{n}}$, Intercept, and $C_{n}$ are the maximal phosphorylation velocity, the apparent affinity constant, the basal phosphorylation rate when the pool size of an effector is zero, and the pool size or concentration of effectors or nutrients, respectively, in equation [4]. The final choice of equation forms was based on goodness of fit statistics or experimental knowledge as explained below. The dephosphorylation reaction was assumed to be mass action throughout:

$$
F_{\text {Protein-PU }}=Q_{\text {Protein- } U} \times k_{P U},
$$

The combined total protein pools were each set to an arbitrary static value of 1 and the differential equations were numerically integrated from a specified initial phosphorylated pool size $\left(i Q_{\text {Protein- } P}\right)$ to predict the phosphorylated and unphosphorylated pool sizes at any point in time:

$$
Q_{\text {Protein }-P}=\int_{\text {Time }(0)}^{\text {Time }(t)} \frac{\mathrm{d} Q_{\text {Protein }-P}}{\mathrm{~d} t}+i Q_{\text {Protein }-P},[6] \text { and }
$$

$$
Q_{\text {Protein- } U}=1-Q_{\text {Protein- } P} .
$$

The mTOR phosphorylation and casein synthesis reactions were initially tested for goodness of fit using linear,

$$
Y=a+(b \times x),
$$

and saturation kinetic representations,

$$
Y=\frac{V_{M a x}}{1+\frac{K m}{[x]}},
$$

through simple linear and nonlinear regression analysis where $a$ and $b$ are the intercept and the slope for the phosphorylation or casein synthesis reactions, respectively, in equation [8]; whereas $V_{M a x}$ and $K m$ are the maximal phosphorylation or casein synthesis rate and the affinity constant, respectively, in equation [9]. In both equations [8] and [9] $x$ refers to the independent variable (i.e., EAA, acetate, glucose, insulin, and intermediate signaling proteins). Based on the resulting statistics and system knowledge, a subset of the equations were retained for further analysis in the dynamic model.

\section{Data}

Data from multiple experiments conducted with MAC-T cells and lactogenic MTS (Appuhamy et al., 2011, 2012, 2014; Appuhamy and Hanigan, 2011; Arriola Apelo et al., 2014b,c) were condensed into a single data set and used for model parameterization. Experiment 1 (Appuhamy et al., 2014) investigated the effects of EAA and acetate or glucose on phosphorylation status of mTOR, AMPK, the downstream signaling proteins $4 \mathrm{eBP} 1$ and eEF2 in both MAC-T cells and MTS, and milk protein synthesis rates in MTS. Experiment 2 (Appuhamy et al., 2012) examined the effects of addition and depletion of individual EAA on the phosphorylation status of mTOR, 4eBP1, eEF2, S6K1, and rpS6 in MAC-T cells and MTS, and on fractional protein synthesis rates in MTS. Experiment 3 (Appuhamy et al., 2011) investigated the effects of EAA and insulin on the phosphorylation status of Akt, mTOR, 4eBP1, S6K1, and eEF2 in both MAC-T cells and MTS, and protein synthesis rates in bovine MTS. Experiment 4 (Appuhamy and Hanigan, 2011) developed a response surface for the effects of EAA $(0,0.35$, 1.00 , and $3.5 \mathrm{mM}$ ) and insulin $(0,5,10$, and $100 \mathrm{ng} /$ $\mathrm{mL}$ ) on the phosphorylation status of Akt, $4 \mathrm{eBP} 1$, and mTOR in MAC-T cells. Experiment 5 (Arriola Apelo 
et al., 2014c) quantified changes in the rate of casein synthesis in response to multiple levels of Ile, Leu, Met, and Thr and their interactions in MTS. Experiment 6 quantified the effects of Ile, Leu, Met, and Thr on the phosphorylation state of mTOR, eEF2, and $\mathrm{rpS6}$ (Arriola Apelo et al., 2014b) in MTS. Phosphorylation was measured by immunoblotting in all studies. Casein synthesis was measured exclusively in MTS by isotopic enrichment of a $\mathrm{pH} 4.6$ precipitate in experiments 1 to 4 and of $\alpha_{S_{1}}$ CN in experiment 5 . We have verified that protein synthesis continues to occur at a constant rate from slaughter up to at least $6 \mathrm{~h}$ in MTS and thus provides data representative of the process in the mammary gland.

Prior to parameterization, protein phosphorylation and casein synthesis data were adjusted to remove variation among studies and blocks within studies (i.e., animal or gel for mammary slice and MAC-T cells, respectively). That was accomplished by fitting the following model to the data:

$$
Y_{i j k}=\mu+S_{i}+B(S)_{i j}+\varepsilon_{i j k}
$$

where $Y_{i j k}$ is the dependent variable, $\mu$ is the mean, $S_{i}$ is the random effect of $i$ th study, $B(S)_{i j}$ is the fixed effect of block within study, and $\varepsilon_{i j k}$ is the residual deviation of the $k$ th observation in the $j$ th block in the $i$ th study from the overall mean which was assumed to be $N \sim(0$, $\left.\sigma^{2}\right)$. Visual inspection of the residual plots was used to verify the above normality and variance assumptions and they were met.

The ACSLX model was constructed using the assumption that phosphorylation ratios (phosphorylated/ total) ranged from 0 to 1 , although immunoblotting data can range almost infinitely due to variation in binding affinity of the 2 different antibodies. Thus, the residuals from the above random effects model, which represented the data after correction for study and block effects, were scaled to an approximate range of 0 to 1 and centered about 0.5 by dividing the residuals by 6 times the estimated standard deviation (i.e., the absolute value of the approximate expected range, and adding a constant, $c$, to all values):

$$
\text { Scaled } Y_{i j k}=\frac{\varepsilon_{i j k}}{6 \times \mathrm{SD}}+c
$$

where $c$ was selected to yield a mean of 0.5.

For casein synthesis rates, the same model was used, but the residuals were not scaled and the overall mean was added back to the residuals to preserve the original units $(\% / \mathrm{h})$ and range:
Table 1. Summary of observed phosphorylation ratios and casein synthesis rates after adjustment for experimental variation and scaling of the phosphorylation data to $6 \mathrm{SD}$ in the interval from 0 to $1^{1}$

\begin{tabular}{lcccc}
\hline Variable $^{2}$ & Mean & SD $^{3}$ & Minimum & Maximum \\
\hline Akt & 0.50 & 0.16 & 0.26 & 0.92 \\
AMPK & 0.50 & 0.16 & 0.18 & 0.93 \\
mTOR & 0.49 & 0.14 & -0.11 & 1.22 \\
S6k1 & 0.50 & 0.14 & 0.02 & 1.12 \\
eEF2 & 0.50 & 0.16 & -0.15 & 1.37 \\
rpS6 & 0.50 & 0.16 & -0.04 & 1.14 \\
4EBP1 & 0.48 & 0.14 & -0.08 & 0.95 \\
Casein synthesis rate & 1.83 & 1.04 & -1.41 & 6.16 \\
\hline
\end{tabular}

${ }^{1}$ Casein data were simply adjusted to remove experimental variance. ${ }^{2} \mathrm{Akt}=$ protein kinase $\mathrm{B} ; \mathrm{AMPK}=\mathrm{AMP}$-activated kinase; $\mathrm{mTOR}=$ mammalian target of rapamycin; eEF2 = eukaryotic elongation factor; eIF2 = eukaryotic initiation factor 2 ; $\operatorname{rpS} 6=$ ribosomal protein $\mathrm{S} 6$; $4 \mathrm{eBP} 1$ = eukaryotic translation initiation factor binding protein 1 .

${ }^{3}$ Standard deviation among observations.

$$
\mu+\varepsilon_{i j k}=Y_{i j k}-\left[S_{i}+B(S)_{i j}\right] .
$$

All experiments had a common positive and negative control; thus, the expected phosphorylation response range was equal across experiments. Descriptive statistics for the final adjusted values for each of the signaling proteins are reported in Table 1 . Table 2 presents a summary of the EAA, acetate, and insulin concentrations used within the collective experiments.

\section{Parameter Estimation}

Model development was guided by previous experimental findings (Appuhamy et al., 2011, 2012, 2014; Appuhamy and Hanigan, 2011; Arriola Apelo et al., 2014b,c), optimization convergence, likelihood based goodness-of-fit methods [Akaike information criterion (AIC) and the Bayesian information criterion (BIC)], error decomposition, and residual plots analysis. Parameter estimation was performed by maximizing the likelihood of a normal density function using the

Table 2. Summary of extracellular AA, insulin, and acetate concentrations across experiments

\begin{tabular}{lcrcc}
\hline $\begin{array}{l}\text { Variable, } \mu \mathrm{mol} / \mathrm{L} \\
\text { (unless noted) }\end{array}$ & Mean & SD & Minimum & Maximum \\
\hline Arg & 506 & 303 & 0.0 & 700 \\
His & 106 & 65 & 0.0 & 150 \\
Lys & 354 & 220 & 0.0 & 500 \\
Phe & 157 & 96 & 0.0 & 220 \\
Trp & 28 & 17 & 0.0 & 40 \\
Val & 323 & 196 & 0.0 & 450 \\
Ile & 210 & 173 & 0.0 & 420 \\
Leu & 226 & 187 & 0.0 & 460 \\
Met & 85 & 70 & 0.0 & 180 \\
Thr & 198 & 194 & 0.0 & 449 \\
Acetate & 0.18 & 0.94 & 0.0 & 5 \\
Insulin, $\mu \mathrm{g} / \mathrm{L}$ & 75 & 42 & 0.0 & 100 \\
\hline
\end{tabular}


Nelder-Mead optimization algorithm (Press, 2007) available in ACSLX (Aegis Technologies Group) where the expected mean is represented by the mechanistic, deterministic, signaling model whereas the residual variation is assumed be $\operatorname{NIID\sim }\left(0, \sigma^{2}\right)$. Residual plots confirmed the normality assumption.

Parameter estimation was undertaken in 2 steps reflecting the 2 subcomponents of the model system: (1) nutrient and hormonal regulation of mTOR using data from MAC-T cells and MTS (410 observations), and (2) mTOR regulation of casein synthesis using data from MTS exclusively (243 observations). For the first component, specific AA, acetate, glucose, and insulin were considered as independent variables. Amino acids affect mTOR directly via an unknown mechanism; acetate and glucose affect mTOR through AMPK; and insulin affects mTOR through Akt. In the second component, mTOR as predicted by the model using the derived parameter estimates became an independent variable that exerted a regulatory effect on casein synthesis through the downstream signaling proteins 4eBP1, S6k1, eEF2, and rpS6.

To define the role of specific EAA as stimulators of mTOR phosphorylation and protein synthesis, efforts were initially focused on evaluating responses to Leu, Ile, Met, and Thr based on previous research findings in our laboratory. In a first experiment aimed at quantifying the effect of the deficiency of each of the 10 EAA on casein synthesis, Appuhamy et al. (2012) observed that these responses were most sensitive to Ile, Leu, Met, and Thr. Based on these findings, experiment 6 was specifically designed to provide sufficient independent variation among them, and we were able to define the surface response of mTOR signaling and protein synthesis to a wide range of supply (Arriola Apelo et al., 2014b,c). Given our observations that these 4 EAA played a predominant role in synthesis regulation, they were evaluated in a stepwise fashion as explained herein.

Once the relationship between mTOR and the 4 AA had been assessed, we inspected the residual plots to check for residual variation in mTOR that may be associated with other EAA (i.e., Arg, His, Lys, Phe, Trp, and Val). Root mean square prediction errors (RMSPE) were calculated and error decomposition analysis was carried out to assess model bias (i.e., adequacy) for each signaling protein and casein synthesis rates (Bibby and Toutenburg, 1977).

Standard error estimates and 95\% confidence intervals for model parameters were obtained through a nonparametric bootstrap (Efron and Tibshirani, 1993; Fox, 2008) implemented in ACSLX. The bootstrap is a method of random resampling of the data with replacement to generate an approximation of the empirical distribution of the parameters as follows (Efron and Tibshirani, 1993). Writing the model in a general manner for the $j$ th observation for the $i$ th response variable (e.g., signaling protein or casein synthesis rate):

$$
\boldsymbol{Y}_{i j}=f\left(\boldsymbol{x}_{i j}, \boldsymbol{\theta}\right)+\boldsymbol{e}_{i j},
$$

where $f$ is the expectation function, $\boldsymbol{x}_{i j}$ is a vector of independent input variables for the $j$ th observation for the $i$ th response variable, $\boldsymbol{\theta}$ is a vector of model parameters, and $\boldsymbol{e}_{i j}$ is a residual term for the $i j$ th observation assumed to be $N I I D \sim\left(0, \sigma^{2}\right)$ (Bates and Watts, 1988); the coefficients of $\boldsymbol{\theta}$ were estimated through maximum likelihood using the original observed data as described above, yielding a matrix of expected values $\left(\hat{\boldsymbol{Y}}_{i j}\right)$ from the inputs $\left(\boldsymbol{x}_{i j}\right)$,

$$
\hat{\boldsymbol{Y}}_{i j}=f\left(\boldsymbol{x}_{i j}, \boldsymbol{\theta}\right),
$$

and a matrix of residuals encompassing each observation in each response variable,

$$
\boldsymbol{e}_{i j}=\boldsymbol{Y}_{i j}-\hat{\boldsymbol{Y}}_{i j} \text {. }
$$

The residuals resulting from the final model fit to the data were subsequently randomly sampled 500 times to populate a bootstrap residuals matrix:

$$
\boldsymbol{e}_{b}^{*}=\left[e_{b 11}^{*}, e_{b 12}^{*}, e_{b 13}^{*}, \ldots, e_{b i j}^{*}\right]^{\prime} .
$$

Each element from the bootstrap residuals matrix, corresponding to the $b$ th bootstrap for the $j$ th observation in the ith response variable, was added to an observation in the same response variable. The process was repeated for each observed response variable yielding a new matrix of bootstrapped observed values,

$$
\boldsymbol{Y}_{b i j}^{*}=\boldsymbol{Y}_{i j}+\boldsymbol{e}_{b i j}^{*}
$$

After repeating this process 500 times, we obtained a vector of 500 bootstrapped observations $\left(\boldsymbol{Y}_{b}^{*}\right)$ values for each response variable:

$$
\boldsymbol{Y}_{b}^{*}=\left[Y_{b 11}^{*}, Y_{b 12}^{*}, \ldots, Y_{b i j}^{*}\right]^{\prime}
$$

The resulting bootstrapped observations were used to refit model parameters, as described above, and the resulting parameters saved in a matrix $\boldsymbol{\theta}_{b}^{*}$. As indicated, this resampling and parameter estimation process was repeated 500 times to create a population of parameters 
that was stored in a matrix. The matrix was used to calculate mean parameter estimates:

$$
\overline{\boldsymbol{\theta}}^{*}=\frac{\sum_{i=1}^{500} \hat{\boldsymbol{\theta}}_{b_{i}}}{500},
$$

and estimates of parameter standard errors $\left[\operatorname{SE}^{*}\left(\overline{\boldsymbol{\theta}}^{*}\right)\right]$ :

$$
\mathrm{SE}^{*}\left(\overline{\boldsymbol{\theta}}^{*}\right)=\sqrt{\frac{\sum_{i=1}^{500}\left(\overline{\boldsymbol{\theta}}_{b_{i}}-\overline{\boldsymbol{\theta}}\right)^{* 2}}{500-1}},
$$

where $\hat{\boldsymbol{\theta}}_{b_{i}}$ is the maximum likelihood estimate from the bth bootstrap ( $i=1$ to 500 runs), and $\overline{\boldsymbol{\theta}}^{*}$ is the parameter estimate computed previously as the average of all 500 bootstraps.

Nonparametric confidence intervals based on the empirical bootstrap sampling distribution (Fox, 2008) were computed to determine whether the parameter approximated or equaled zero. The 500 bootstrap parameter estimates were ranked numerically and the $95 \%$ percentile interval was computed as

$$
\overline{\boldsymbol{\theta}}_{\text {Lower }}^{*}<\boldsymbol{\theta}<\overline{\boldsymbol{\theta}}_{\mathrm{Upper}}^{*},
$$

where $\overline{\boldsymbol{\theta}}_{\text {Lower }}^{*}$ and $\overline{\boldsymbol{\theta}}_{\mathrm{Upper}}^{*}$ are the parameter values at the 5th and 95th percentiles, respectively.

\section{Global Sensitivity Analysis}

A global sensitivity analysis (GSA; Saltelli et al., 1999) was performed to assess the sensitivity of protein phosphorylation and casein synthesis predictions to inputs and parameter estimates. The physiological range of insulin, acetate, and EAA concentrations were the inputs used (Table 8). Model parameters tested included all of those fitted to data in the final model. The GSA computes the partial change (i.e., sensitivity coefficients) in protein phosphorylation and casein synthesis rates with respect to the marginal change in the model inputs or parameters. Sensitivity coefficients (SC) and their sum represent the proportion of the total variation associated with the parameter taking a value between 0 and 1 . The larger the value of the coefficient, the more influence a model input or parameter has on the model outcome. The GSA was conducted in 2 different parts, the first part assessed sensitivity to nutrient inputs (Table 8); whereas, the second assessed sensitivity to model parameters (Table 8). Insulin, acetate, Leu, and Ile concentrations were set to range from 0.34 to $10.4 \mu \mathrm{g} / \mathrm{L}$ (Blum et al., 1973), 1.02 to 3.02 $\mathrm{m} M, 30$ to $243 \mu M$, and 42 to $254 \mu M$, respectively, based on previous reports of in vivo blood concentrations (Hanigan et al., 2002). Model parameters assessed included those listed in Table 8. Random sampling was from a uniform distribution within the specified ranges.

\section{RESULTS AND DISCUSSION}

\section{Exploratory Univariate Static Analysis}

Univariate relationships between Akt and insulin; AMPK and glucose and acetate; mTOR and specific EAA (i.e., Leu, Ile, Met and Thr), Akt, and AMPK; and casein synthesis and 4eBP1, eEF2, S6k1, rpS6, or mTOR were evaluated to determine whether linear or saturating functions would fit the protein phosphorylation and casein synthesis data better (Table 3) before undertaking the dynamic multivariate modeling. Both linear and M-M regression equations were compared based on the AIC and the BIC between a constant (i.e., intercept only) and full models, and significance of parameters being different from zero at $\alpha=0.2$. A smaller AIC or BIC indicates a better fit to the data, and the later method penalizes a larger number of parameters. Functions were fitted to data by minimization of least or weighted least squares using the $\mathrm{lm}$ and nls functions of the Stats package of R (R Core Team, 2014).

Phosphorylation of Akt was better described by an $\mathrm{M}-\mathrm{M}$ equation form $(\mathrm{AIC}=-63, \mathrm{BIC}=-57)$ than $\mathrm{a}$ constant $(\mathrm{AIC}=-22, \mathrm{BIC}=-19)$ or a linear $(\mathrm{AIC}=$ -58 , BIC $=-54$, Table 3) function; hence, this representation was retained in the final dynamic model. Thus, the linear model of Appuhamy and Hanigan (2011) was modified to represent the effects of insulin on Akt phosphorylation $\left[F_{\mathrm{Akt}-U P}\right.$, arbitrary units $(\mathbf{A U}) / \mathrm{h}]$ as a saturable process:

$$
F_{\text {Akt-UP }}=\left[\frac{V_{\text {Akt-UP }}}{1+\left(K_{\text {Insulin-Akt }} / C_{\text {Insulin }}\right)}\right]+\mathrm{Akt}_{\text {Intercept }},
$$

where $C_{\text {Insulin }}$ represented the extracellular concentration of insulin $(\mu \mathrm{g} / \mathrm{mL}), K_{\text {Insulin-Akt }}$ represented the apparent affinity constant $(\mu \mathrm{g} / \mathrm{mL})$, and $V_{\text {Akt-UP }}$ represented the maximal phosphorylation velocity $(\mathrm{AU} / \mathrm{h})$. An intercept $\left(\mathrm{Akt}_{\text {Intercept }}, \mathrm{AU} / \mathrm{h}\right)$ was included as experimentally measured basal levels of Akt phosphorylation were greater than 0 in the absence of insulin.

Phosphorylation of AMPK was best described by a linear function compared with the nonlinear form. Only acetate appeared to have some inhibitory effect and 
Table 3. Univariate regression analysis of independent variable effects on mammalian target of rapamycin (mTOR) phosphorylation and casein synthesis rates

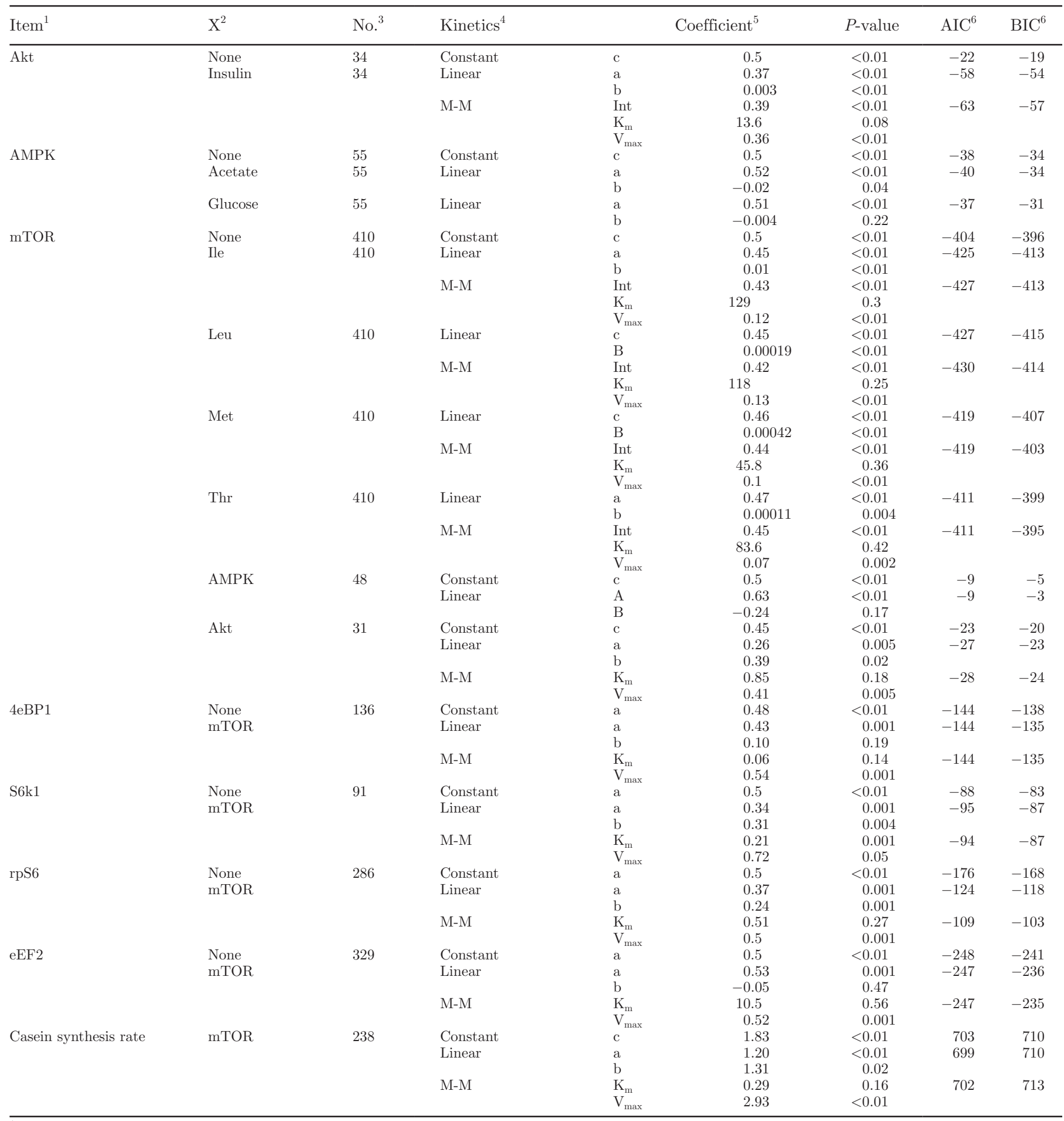

${ }^{1}$ Akt $=$ protein kinase $\mathrm{B} ; \mathrm{AMPK}=\mathrm{AMP}$-activated kinase eEF2 = eukaryotic elongation factor; eIF2 = eukaryotic initiation factor $2 ;$ rpS6 = ribosomal protein $\mathrm{S} 6$; 4eBP1 = eukaryotic translation initiation factor binding protein 1 .

${ }^{2} \mathrm{X}=$ independent variable.

${ }^{3}$ No. of observations.

${ }^{4}$ Type of equation. Linear: first-order polynomial, M-M: Michaelis-Menten.

${ }^{5}$ Coefficients: $\mathrm{c}=$ constant, $\mathrm{a}=$ intercept, $\mathrm{b}=$ slope, Int = intercept for $\mathrm{M}-\mathrm{M}, \mathrm{K}_{\mathrm{m}}=$ affinity constant for $\mathrm{M}-\mathrm{M}, \mathrm{V}_{\max }=\operatorname{maximal}$ velocity for M-M.

${ }^{6} \mathrm{AIC}=$ Akaike information criterion; BIC $=$ Bayesian information criterion. 
gave only a slightly better fit (slope $=-0.02, P=0.04$, $\mathrm{AIC}=-40, \mathrm{BIC}=-34$ ) compared with a constant $(\mathrm{AIC}=-38, \mathrm{BIC}=-34)$ or a function of glucose (slope $=-0.004, \mathrm{AIC}=-37, \mathrm{BIC}=-31$, Table 3 ); thus, only acetate was included in the final dynamic model as a mass action inhibitor $\left(F_{\mathrm{AMPK}-U P}, \mathrm{AU} / \mathrm{h}\right)$ with acetate inhibition:

$$
\begin{gathered}
F_{\text {AMPK- } U P}=\left[k_{\text {AMPK- } U P}-J_{\text {Acetate-AMPK }} C_{\text {Acetate }}\right] \\
\times Q_{\text {AMPK- } U},
\end{gathered}
$$

where $C_{\text {Acetate }}$ represents the extracellular concentration of acetate $(\mu \mathrm{mol} / \mathrm{L}), k_{\mathrm{AMPK}-U P}$ is the mass action constant, $J_{\text {Acetate-AMPK }}$ is the inhibition constant for acetate, and $Q_{\mathrm{AMPK}-U}$ is the pool size of unphosphorylated AMPK to which the overall phosphorylation reaction rate is proportional.

Based on the AIC, phosphorylation of mTOR was slightly better described by an M-M function of Leu $(\mathrm{AIC}=-430)$ than a constant $(\mathrm{AIC}=-404)$ or a linear function of Leu (AIC $=-427)$, or a linear or M-M function of Ile, Met, or Thr, even though the affinity constant for Leu was not significant at $\alpha=0.2$ $(P=0.25$, Table 3$)$. Because experimentally we also observed that the average phosphorylative effect of these EAA reaching saturation at AA concentrations $\sim 500 \mu M$ (Arriola Apelo et al., 2014b), we decided to retain an M-M representation for further model building. Whereas the inhibitory effect of AMPK on mTOR could not be described nonlinearly due to lack of convergence to a solution, we noted a weak linear effect (slope $=-0.24, P=0.17, \mathrm{AIC}=-9, \mathrm{BIC}=$ -3 , Table 3); however, representation of such an effect in the model resulted in a slightly worse fit than a constant model $(\mathrm{AIC}=-9, \mathrm{BIC}=-5)$. Additionally, the stimulatory effect of Akt on mTOR was described with similar goodness of fit by an M-M $(\mathrm{AIC}=-27$, $\mathrm{BIC}=-23)$ and a linear function $(\mathrm{AIC}=-28, \mathrm{BIC}=$ $-24)$, but the linear slope was significant at $\alpha=0.05$ whereas the M-M affinity constant was only so at $\alpha$ $=0.2$ (Table 3 ). Both fits were better than a constant representation $(\mathrm{AIC}=-23, \mathrm{BIC}=-20)$. Downstream, phosphorylated mTOR affects phosphorylation of 4eBP-1 and S6k1, the latter of which affects protein synthesis through phosphorylation of the ribosomal protein rpS6 and dephosphorylation of the elongation factor eEF2.

With respect to $4 \mathrm{eBP} 1$, the goodness of fit was better for a constant model $(\mathrm{BIC}=-138)$ compared with both a linear $(\mathrm{BIC}=-135)$ and saturating $(\mathrm{BIC}=$ $-135)$ function of phosphorylated mTOR, even though the respective slope $(P=0.19)$ and affinity constant $(P$ $=0.14$ ) were different from 0 at $\alpha=0.2$ (Table 3). For
S6k1, both linear (slope $=0.31, P=0.004)$ and saturation effects of phosphorylated mTOR $(K m=0.21, P=$ 0.001 ) were observed, and both forms presented a better fit $(\mathrm{BIC}=-87)$ than a constant model $(\mathrm{BIC}=-83$; Table 3). In the case of rpS6, both the linear (AIC = $-124, \mathrm{BIC}=-118)$ and saturating $(\mathrm{AIC}=-109, \mathrm{BIC}$ $=-103)$ functions of phosphorylated mTOR resulted in a largely poorer fit than the constant model (AIC = $-176, \mathrm{BIC}=-168$, Table 3). Finally, use of phosphorylated $\mathrm{mTOR}$ as a predictor of eEF2 phosphorylation in both linear and saturating functions was not useful as indicated by a slope $(P=0.47)$ and affinity constant $(P$ $=0.56)$ that were not different from 0 at $\alpha=0.2$, and $\mathrm{AIC}$ and BIC values greater than those of a constant model indicating a poorer model fit (Table 3). Therefore, the only signaling protein downstream of mTOR for which the data appeared to support a relationship with phosphorylated mTOR was S6k1.

Based on the AIC or BIC, representing casein synthesis as a linear function of phosphorylated mTOR or a linear or saturating function of S6k1, provided a better fit than the corresponding constant representations (Table 3). Given the statistically significant mTOR/ S6k1 signaling effects, and because we experimentally observed that the additive effect of Leu, Ile, Met, and Thr on casein synthesis was saturable (Arriola Apelo et al., 2014c), we preserved only the M-M representation for further testing in the final dynamic model. Meantime, $4 \mathrm{eBP} 1, \mathrm{rpS} 6$, or eEF2 in linear or saturating form did not contribute to explain casein synthesis data any better in the exploratory univariate analysis (data not shown). This signaling cascade was further evaluated in the exploratory multivariate dynamic analysis following below to decide whether inclusion of additional elements downstream of mTOR were warranted.

\section{Exploratory Multivariate Dynamic Analysis}

Based on our previous experimental evidence and results from the above univariate static exploratory analysis, alternative multivariate representations of mTOR signaling and control of casein synthesis were considered within the dynamic ACSLX model (Table 4). Improvement in goodness of fit measures (AIC and $\mathrm{BIC}$ ) between full and reduced models were the primary criteria for the evaluations. Table 4 shows the RMSPE and its partition as well as the total number of observations used to parameterize the preliminary multivariate equations.

The mTOR phosphorylation was better predicted when the effect of Leu was included (AIC $=-781$, BIC $=-769)$ than when an intercept only model was fit $(\mathrm{AIC}=-752, \mathrm{BIC}=-756$; Table 4$)$. Based on AIC, adding the effect of Ile slightly improved the fit (AIC = 
Table 4. Multivariate regression analysis of independent variable effects on signaling proteins and casein synthesis rates

\begin{tabular}{|c|c|c|c|c|c|c|}
\hline Item $^{1}$ & Kinetics $^{2}$ & $\mathrm{AIC}^{3}$ & $\mathrm{BIC}^{3}$ & $\mathrm{RMSPE}^{4}$ & $\mathrm{MB}^{5}$ & $\mathrm{SB}^{6}$ \\
\hline \multicolumn{7}{|l|}{ mTOR } \\
\hline $\mathrm{C}$ & Constant & -752 & -756 & 29.7 & 0 & 0 \\
\hline $\mathrm{C}+\mathrm{Leu}$ & $\mathrm{M}-\mathrm{M}$ & -781 & -769 & 28.6 & 0 & 0 \\
\hline $\mathrm{C}+\mathrm{Leu}+\mathrm{Ile}$ & M-M & -784 & -769 & 28.4 & 0 & 0 \\
\hline $\mathrm{C}+\mathrm{Leu}+\mathrm{Ile}+$ Met & M-M & -785 & -765 & 28.3 & 0 & 0 \\
\hline $\mathrm{C}+\mathrm{Leu}+\mathrm{Ile}+$ Insulin & M-M & -748 & -728 & 29.6 & 0 & 0 \\
\hline $\mathrm{C}+\mathrm{Leu}+\mathrm{Ile}+$ Insulin + AMPK & $\mathrm{M}-\mathrm{M}$ & -781 & -757 & 28.4 & 0 & 0 \\
\hline \multicolumn{7}{|l|}{ Casein synthesis rate } \\
\hline $\mathrm{C}$ & Constant & 504 & 507 & 56.8 & 0 & 0 \\
\hline $\mathrm{C}+\mathrm{mTOR}$ & M-M & 488 & 495 & 54.7 & 0 & 0.2 \\
\hline $\mathrm{C}+\mathrm{S} 6 \mathrm{k} 1$ & M-M & 488 & 495 & 54.7 & 0 & 0.2 \\
\hline $\mathrm{C}+4 \mathrm{eBP} 1$ & $\mathrm{M}-\mathrm{M}$ & 494 & 501 & 55.4 & 0 & 0 \\
\hline $\mathrm{C}+\mathrm{S} 6 \mathrm{k} 1+4 \mathrm{eBP} 1$ & M-M & 494 & 505 & 55.2 & 0 & 0.4 \\
\hline $\mathrm{C}+4 \mathrm{ebp} 1+\mathrm{rpS} 6+\mathrm{eEF} 2$ & $\mathrm{M}-\mathrm{M}$ & 496 & 510 & 55.2 & 0 & 0.5 \\
\hline
\end{tabular}

${ }^{1} \mathrm{C}=$ constant (i.e., intercept only model); AMPK = AMP-activated kinase; $\mathrm{mTOR}=$ mammalian target of rapamycin; eEF2 = eukaryotic elongation factor; $\mathrm{rpS} 6=$ ribosomal protein $\mathrm{S} 6 ; 4 \mathrm{eBP} 1=$ eukaryotic translation initiation factor binding protein 1; S6k1 = protein S6 kinase 1.

${ }^{2}$ Type of equation. Constant: intercept only model; M-M: Michaelis-Menten equation.

${ }^{3} \mathrm{AIC}=$ Akaike information criterion; $\mathrm{BIC}=$ Bayesian information criterion

${ }^{4} \mathrm{RMSPE}=$ root mean square prediction error.

${ }^{5} \mathrm{MB}=$ mean bias.

${ }^{6} \mathrm{SB}=$ slope bias.

-784 , BIC $=-769)$ compared with a Leu model only (Table 4), and adding the effect of Met did not provide any additional improvements $(\mathrm{AIC}=-785, \mathrm{BIC}=$ -765); addition of Thr resulted in convergence problems. The order of inclusion of these 4 EAA was based on the ranking of their $\mathrm{AIC}$ and $\mathrm{BIC}$ in the univariate exploratory analysis, where Leu was best and Thr was worst. Adding the saturable, regulatory effect of insulin via Akt onto mTOR regulation did not produce a better fit $(\mathrm{AIC}=-748, \mathrm{BIC}=-728)$ compared with the intercept only model $(\mathrm{AIC}=-781, \mathrm{BIC}=-769)$ or the model containing Leu and Ile effects $(\mathrm{AIC}=-784$, BIC $=-769$; Table 4 ), even though an M-M representation did improve the fit to phosphorylated mTOR data in the univariate setting as described above. Nevertheless, as the role of insulin on mTOR and protein synthesis regulation is well known (Suryawan et al., 2009), we preserved this element of the model to identify research needs from a subsequent sensitivity analysis. Similarly, addition of AMPK to the mTOR model with Leu and Ile regulation did not result in improvements in the model fit $(\mathrm{AIC}=-781, \mathrm{BIC}=-757)$ compared with including only Leu and Ile $(\mathrm{AIC}=-784, \mathrm{BIC}=-769$; Table 4 ), but this addition was maintained for the purposes of conducting sensitivity analysis. All mTOR representations considered had RMSPE between 28 and $31 \%$ with no mean or slope bias (Table 4). Therefore, as a result of previous experimental work where EAA and insulin effects on mTOR were saturating and independent (Appuhamy et al., 2011, 2012; Arriola Apelo et al., 2014b) and the slightly better goodness of fit obtained in the univariate and multivariate analysis with an M-M function, the original mTOR kinase reaction representation (Appuhamy and Hanigan, 2011) was converted to an M-M form with simultaneous stimulatory effects of phosphorylated Akt $\left(Q_{\text {Akt }-P}\right)$, Leu $\left(C_{\text {Leu }}\right)$, and Ile $\left(C_{\text {Ile }}\right)$, and the inhibitory effect of AMPK $\left(Q_{\text {AMPK-P }}\right)$ :

$$
\begin{aligned}
& F_{\text {mTOR-UP }}=\left(\frac{V_{\text {mTOR-UP }}}{1+\left[\frac{K_{\text {Akt-TOR }}}{Q_{\text {Akt }-P}}+\frac{K_{\text {Leu-TOR }}}{C_{\text {Ile }}}+\frac{K_{\text {Ile-TOR }}}{C_{\text {lle }}}+\frac{Q_{\text {AMPK- }-P}}{J_{\text {AMPK-TOR }}}\right]}\right) \\
& + \text { mTOR }_{\text {Intercept }},
\end{aligned}
$$

where $K_{\text {AKT-TOR }}, K_{\text {Leu-TOR }}, K_{\text {Ile-TOR }}$, and $J_{\text {AMPK-TOR }}$ are the respective affinity and inhibition constants in $\mathrm{AU}$ for signaling proteins and micromoles per liter for AA.

Following from the univariate analysis, using a saturable response equation for casein synthesis in response to phosphorylated mTOR resulted in an improved fit $(\mathrm{AIC}=488, \mathrm{BIC}=495)$ compared with an intercept only model $(\mathrm{AIC}=504, \mathrm{BIC}=507)$ and reduced the prediction error by $\sim 2 \%$ (Table 4 ). To find out whether the casein synthesis data could be better represented by a more detailed description downstream of mTOR, the role of S6k1, eEF2, rpS6, and 4eBP1 on casein synthesis rates was assessed. A similar goodness of fit (e.g., AIC, BIC, and RMSPE) was obtained when a 
saturable response to phosphorylated $\mathrm{S} 6 \mathrm{k} 1$ was added to the intercept only model $(\mathrm{AIC}=488, \mathrm{BIC}=495$; Table 4) compared with considering phosphorylated mTOR only $(\mathrm{AIC}=488, \mathrm{BIC}=495)$. Adding $4 \mathrm{eBP} 1$ as a saturable effect was better $(\mathrm{AIC}=494, \mathrm{BIC}=501$; Table 4) than the constant model $(\mathrm{AIC}=504, \mathrm{BIC}$ $=507$ ), but was not as good a fit as the S6k1 model. Adding a saturating effect of both $4 \mathrm{eBP} 1$ and S6k1 provided no additional improvement in fit $(\mathrm{AIC}=494$, $\mathrm{BIC}=505$; Table 4$)$ as compared with their individual representations. Following a hierarchical order in the mTOR signaling cascade, addition of the effect of eEF2 and rpS6 to the 4eBP1/S6k1 saturating model also did not improve the fit $(\mathrm{AIC}=496, \mathrm{BIC}=510$, Table 4$)$. As mTOR modulates protein synthesis both through S6k1 and 4eBP1, we decided to retain the model considering mTOR as a direct saturating effector; as such, a representation implicitly encompasses both signaling paths instead of choosing only S6k1 or 4eBP1, both of which improved the fit partially on their own but not together. All casein synthesis representations that were considered yielded high RMSPE of 55 to $58 \%$ (Table 4).

The final M-M representation of fractional casein synthesis $\left(F_{\text {CaseinSyn }}, \% / \mathrm{h}\right)$ was

$$
F_{\text {CaseinSyn }}=\frac{V_{\text {CaseinSyn }}}{1+\left[\frac{K_{\text {mTOR-Casein }}}{Q_{\text {mTOR }-P}}\right]},
$$

where $K_{\mathrm{mTOR}-\mathrm{Casein}}$ and $Q_{\mathrm{mTOR}-P}$ represented the affinity constant for mTOR (AU) and the pool size of phosphorylated mTOR (AU), respectively.

As no data were available to estimate the dephosphorylation (i.e., phosphatase reaction) rate constants of these cycling systems, the rate constant $\left(k_{P U}\right)$ for the phosphatase reactions were set to values ensuring less than $25 \%$ of the pool was turning over in a single integration step to maintain model stability during numerical integration. These assumed rates were maintained for the entirety of the work. Dephosphorylation reactions for Akt, AMPK, and mTOR were defined, respectively, as

$$
\begin{gathered}
F_{\mathrm{Akt}-P U}=k_{\mathrm{Akt}-P U} \times Q_{\mathrm{Akt}-P}, \\
F_{\mathrm{AMPK}-P U}=k_{\mathrm{AMPK}-P U} \times Q_{\mathrm{AMPK}-P},[26] \text { and } \\
F_{\mathrm{mTOR}-P U}=k_{\mathrm{mTOR}-P U} \times Q_{\mathrm{mTOR}-P .} .
\end{gathered}
$$

The resulting reduced model thus resulted in a dynamic system of 6 differential equations corresponding to 6 state variables, each representing the rate of change in the phosphorylated and dephosphorylated pools of Akt,
AMPK, and mTOR (Figure 2). Thus, based on the generic equations [1] and [2], and using equations [21], [22], and [23], the instantaneous rate of change in the phosphorylated and unphosphorylated pools of Akt, AMPK, and mTOR can be computed as the difference between the respective kinase (equations[21], [22], and [23]) and phosphatase (equations [25], [26], and [27]) reactions:

$$
\begin{gathered}
\frac{\mathrm{d} Q_{\mathrm{Akt}-P}}{\mathrm{~d} t}=F_{\mathrm{Akt}-U P}-F_{\mathrm{Akt}-P U}, \\
\frac{\mathrm{d} Q_{\mathrm{Akt}-U}}{\mathrm{~d} t}=-\mathrm{d} Q_{\mathrm{Akt}-P}, \\
\frac{\mathrm{d} Q_{\mathrm{AMPK}-P}}{\mathrm{~d} t}=F_{\mathrm{AMPK}-U P}-F_{\mathrm{AMPK}-P U}, \\
\frac{\mathrm{d} Q_{\mathrm{AMPK}-U}}{\mathrm{~d} t}=-\mathrm{d} Q_{\mathrm{AMPK}-P}, \\
\frac{\mathrm{d} Q_{\mathrm{mTOR}-P}}{\mathrm{~d} t}=F_{\mathrm{mTOR}-U P}-F_{\mathrm{mTOR}-P U},[32] \text { and } \\
\frac{\mathrm{d} Q_{\mathrm{mTOR}-U}}{\mathrm{~d} t}=-\mathrm{d} Q_{\mathrm{mTOR}-P} .
\end{gathered}
$$

And from equations [6], [7], [12], [14], and [16], the signaling protein pool sizes for Akt, AMPK, and mTOR are computed respectively as:

$$
\begin{gathered}
Q_{\mathrm{Akt}-P}=\int_{\operatorname{Time}(0)}^{\operatorname{Time}(t)} \frac{\mathrm{d} Q_{\mathrm{Akt}-P}}{\mathrm{~d} t}+i Q_{\mathrm{Akt}-P}, \\
Q_{\mathrm{Akt}-P}=1-Q_{\mathrm{Akt}-P}, \\
Q_{\mathrm{AMPK}-P}=\int_{\operatorname{Time}(0)}^{\operatorname{Time}(t)} \frac{\mathrm{d} Q_{\mathrm{AMPK}-P}}{\mathrm{~d} t}+i Q_{\mathrm{AMPK}-P}, \\
Q_{\mathrm{AMPK}-P}=1-Q_{\mathrm{AMPK}-P}, \\
Q_{\mathrm{mTOR}-P}=\int_{\operatorname{Time}(0)}^{\operatorname{Time}(t)} \frac{\mathrm{d} Q_{\mathrm{mTOR}-P}}{\mathrm{~d} t}+i Q_{\mathrm{mTOR}-P}, \\
Q_{\mathrm{mTOR}-U}=1-Q_{\mathrm{mTOR}-P .} .
\end{gathered}
$$

The set of abbreviations for all of the terms in the above equations are given in Table 5 and a scheme with steady state flux rates (i.e., phosphorylation rates equal 
dephosphorylation rates) and pool sizes for average physiological extracellular concentrations of Leu, Ile, insulin, and acetate for the solved model in the reference state is presented in Figure 2. This information al- lows the interested reader to have an idea of the fluxes and relative pool sizes when this system is at steady state under the experimental conditions in which we gathered the data.

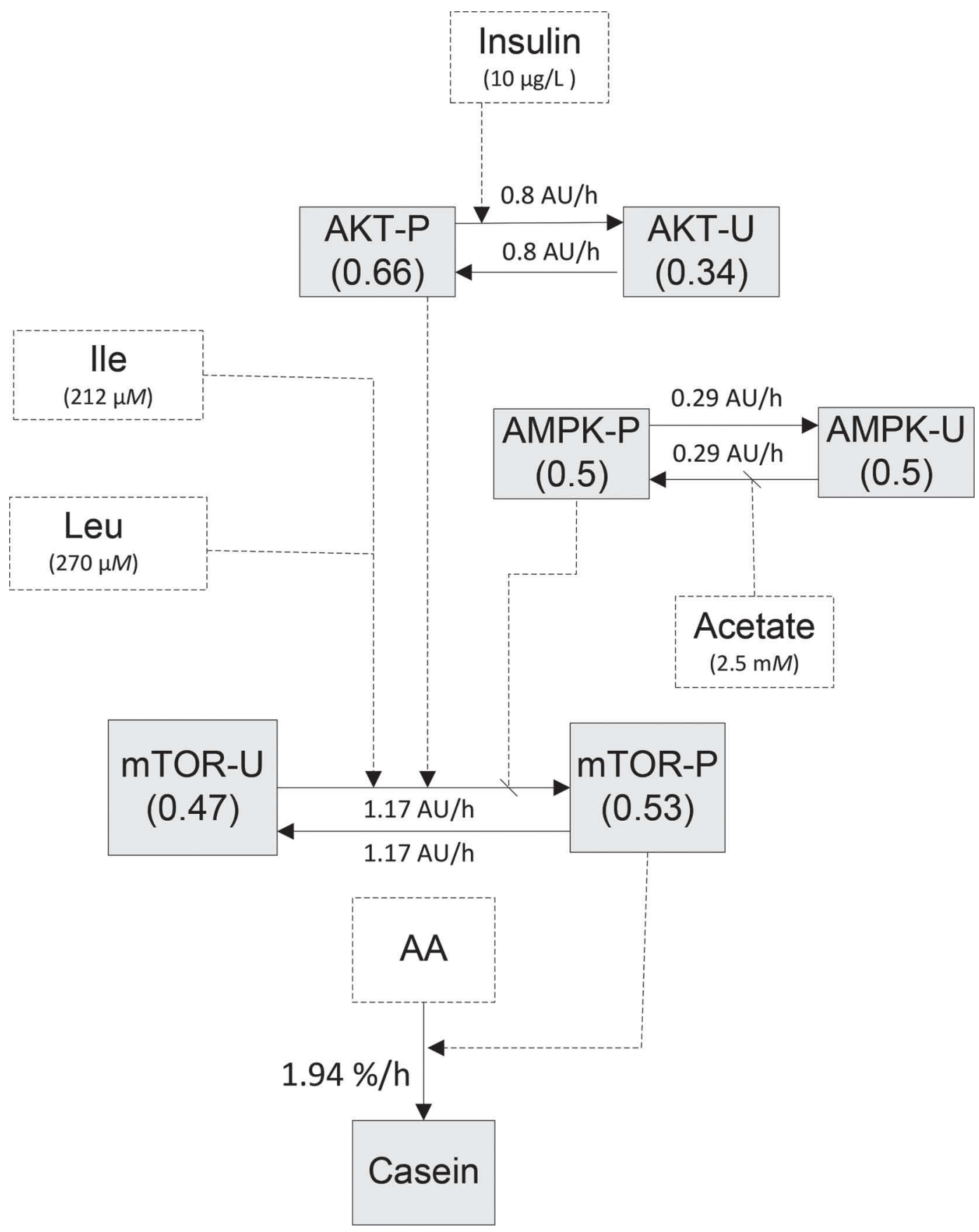

Figure 2. Reduced parameterized model of protein synthesis regulation through mTOR signaling at steady state. Boxes enclosed by dashed lines are model inputs; boxes enclosed by solid lines are state variables; solid arrows denote fluxes; dashed arrows denote regulation with an arrow line endpoint indicating a positive regulator and an angled bar endpoint denoting an inhibitory regulator. Phoshorylated signaling proteins are denoted by a terminal $\mathrm{P}$ in the name and unphosphorylated forms by a terminal $\mathrm{U}$ in the name. Fluxes are denoted in arbitrary units per hour and casein synthesis rates in percent per hour. The reference extracellular conditions to yield the above steady state representation were insulin at $10 \mu \mathrm{g} / \mathrm{L}$, Ile and at Leu at 212 and $270 \mu M$, respectively, and acetate at $2.5 \mathrm{mM}$. Akt = protein kinase B; AMPK = AMP-activated protein kinase; $\mathrm{mTOR}=$ mammalian target of rapamycin. 
Table 5. Model nomenclature and abbreviations

\begin{tabular}{ll}
\hline Abbreviation & Description \\
\hline$V_{\text {Akt-UP }}$ & Maximal velocity for the Akt (protein kinase B) phosphorylation reaction \\
$K_{\text {Insulin-Akt }}$ & Insulin affinity constant for the Akt phosphorylation reaction \\
Akt Intecept & Basal rate of Akt phosphorylation \\
$k_{\text {AMPK-UP }}$ & Mass action rate constant for the AMPK (AMP activated protein kinase) \\
$J_{\text {Acetate-AMPK }}$ & phosphorylation reaction \\
mTOR $_{\text {Intercept }}$ & Mass action inhibition rate constant for acetate in the AMPK phosphorylation reaction \\
$V_{\text {mTOR-UP }}$ & Maximal of miolocity of Akt phosphorylation \\
$K_{\text {Akt-TOR }}$ & Akt affinity constant for the mTOR phosphorylation reaction \\
$K_{\text {Ile-TOR }}$ & Isoleucine affinity constant for the mTOR phosphorylation reaction \\
$K_{\text {Leu-TOR }}$ & Leucine affinity constant for the mTOR phosphorylation reaction \\
$J_{A M P K-T O R}$ & AMPK inhibition constant for the mTOR phosphorylation reaction \\
$V_{\text {CaseinSyn }}$ & Maximal velocity for casein synthesis \\
$K_{\text {mTOR-Casein }}$ & mTOR affinity constant for the casein synthesis reaction \\
$C_{x}$ & Extracellular concentration of nutrient x \\
$F_{\text {Protein-UP }}$ & Flux for unphosphorylated signaling protein being converted to a phosphorylated form \\
$F_{\text {CaseinSyn }}$ & Casein synthesis flux \\
$Q_{\text {Protein-P }}$ & The pool size of a phosphorylated signaling protein \\
\hline
\end{tabular}

Bootstrap parameter estimate values, standard errors, coefficient of variation, and confidence intervals for our final reduced model are presented in Table 6. Initial work yielded a mean bias in the estimated phosphorylated Akt pool size, which led us to include an intercept in the phosphorylation reaction, indicating that even at very low concentrations of insulin, the basal reaction velocity proceeded at $\sim 50 \%$ (i.e., $\mathrm{Akt}_{\text {Intecept }}=0.41$ ) of the maximum (i.e., $\mathrm{Akt}_{\text {Intecept }}+V_{\mathrm{Akt}-U P}=0.86$, Table $6)$. Also, the affinity constant $\left(K_{\text {Insulin-Akt }}\right)$ indicates that half maximal phosphorylation of Akt occurs at $\sim 15.4$ $\mu \mathrm{g} / \mathrm{L}$, which indicates the response could be expected to be in the linear portion of the curve given in vivo blood insulin concentrations ranging between approximately
0.28 and $10.4 \mu \mathrm{g} / \mathrm{L}$ (Blum et al., 1973, Juchem et al., 2004). Whereas, none of the confidence intervals for the 3 parameters defining Akt phosphorylation overlapped 0 , the affinity constant $\left(K_{\text {Insulin-Akt }}\right)$ was poorly defined with a coefficient of variation of $52 \%$ and a confidence interval from 5.82 to $32.7 \mu \mathrm{g} / \mathrm{L}$ (Table 6 ). The final Akt representation had the smallest RMSPE $(16.8 \%)$ of all signaling proteins with no mean or slope bias, indicating an adequate fit despite having been parameterized from the smallest number of observations (Table 7).

The sensitivity of Akt to insulin was strong, at 0.97 (Table 8), as one would expect of a representation of a well-established signaling pathway (Lizcano and Alessi, 2002). Figure 3a displays the predicted response in

Table 6. Model parameter estimates, standard errors, and confidence intervals

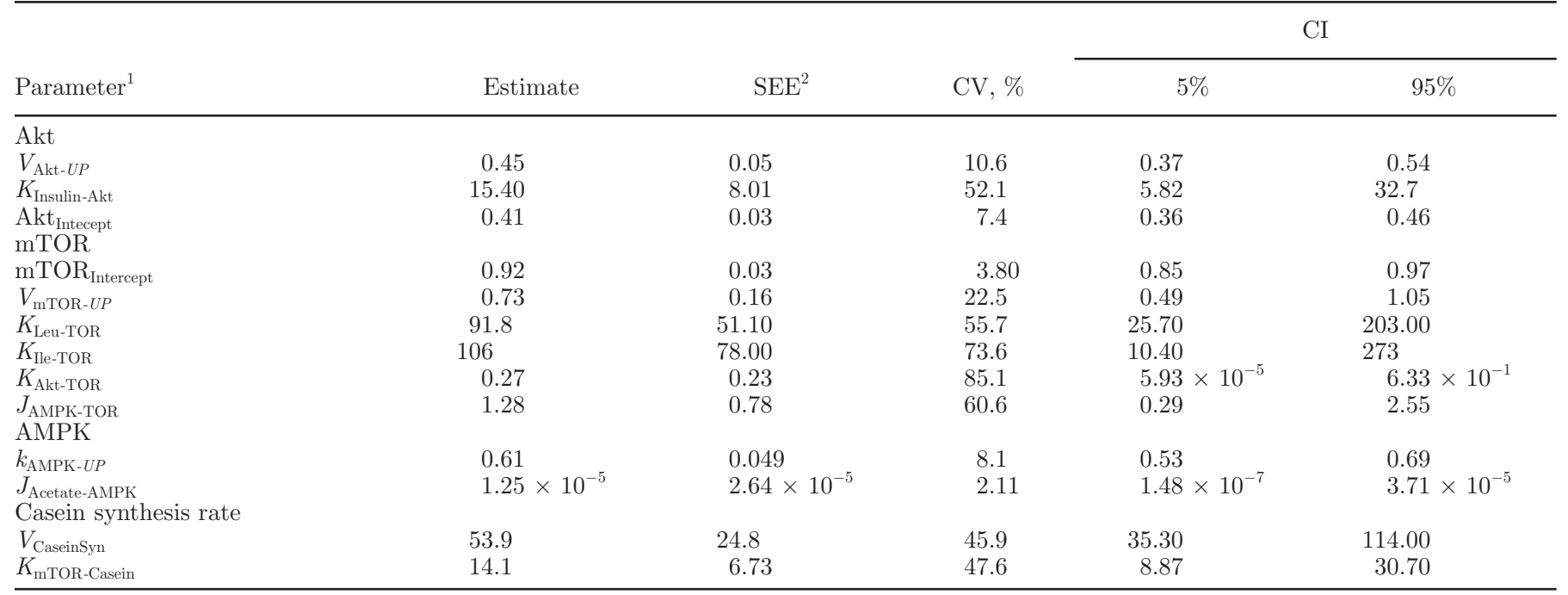

${ }^{1}$ Abbreviations from Table 5.

${ }^{2} \mathrm{SEE}=$ standard error of the estimate. 
Table 7. Model prediction error, decomposition, and sample size for each dependent variable

\begin{tabular}{|c|c|c|c|c|c|}
\hline Variable $^{1}$ & RMSPE, ${ }^{2} \%$ & $\mathrm{MB},{ }^{3}$ & $\%$ of RMSPE & $\mathrm{SB},{ }^{4} \%$ of RMSPE & Observations, no. \\
\hline mTOR & 28.4 & & 0.0 & 0.2 & 410 \\
\hline AMPK & 33.0 & & 0.0 & 7.4 & 55 \\
\hline
\end{tabular}

${ }^{1} \mathrm{Akt}=$ protein kinase $\mathrm{B} ; \mathrm{mTOR}=$ mammalian target of rapamycin; AMPK = AMP activated protein kinase.

${ }^{2} \mathrm{RMSPE}=$ root mean square prediction error as a percent of the observed mean.

${ }^{3} \mathrm{MB}=$ mean bias.

${ }^{4} \mathrm{SB}=$ slope bias.

the pool size of phosphorylated Akt at varying insulin concentrations. As blood insulin increased from 0 to 10 $\mu \mathrm{g} / \mathrm{L}$, phosphorylated Akt increased by roughly $30 \%$, from $\sim 0.35$ to 0.5 . With respect to the parameters, Akt was similarly sensitive to the basal $\left(\mathrm{Akt}_{\text {Intercept }}, \mathrm{SC}=\right.$ $0.30)$ and maximal phosphorylation velocity ( $V_{\text {Akt- } U P}$, $\mathrm{SC}=0.27)$ parameters; whereas the system was minimally sensitive $(\mathrm{SC}=0.03)$ to the affinity constant for insulin $\left(K_{\text {Insulin-Akt }}\right.$; Table 8$)$. It thus appears that to improve our knowledge of Akt phosphorylation in the mammary system, one should look at animal intrinsic factors (e.g., milk production level) that affect basal and maximal phosphorylation as an overall measure of insulin sensitivity.

The AMPK phosphorylation reflects the energy status of mammary cells and inhibits ATP-consuming processes such as protein synthesis via mTOR under nutrient restriction (Viollet et al., 2010; Burgos et al., 2013). Evidence exists that acetate and glucose are the primary oxidative energy substrates in the cow's whole body accounting for $\sim 33$ and $30 \%$ of the $\mathrm{CO}_{2}$ yield from whole body, respectively (Bickerstaffe et al., 1974); whereas, in the mammary cell, acetate would be the major $\mathrm{CO}_{2}$ contributor, accounting for $45 \%$ of the total yield compared with only $8.3 \%$ from glucose (Hanigan et al., 2001). Therefore, one would expect at least acetate to be inhibitory with respect to AMPK phosphorylation in the mammary system. Here, acetate had a small linear effect on phosphorylation of AMPK $\left(J_{\text {Acetate-AMPK }}=1.25 \times 10^{-5} \mathrm{mmol} / \mathrm{L}\right.$, Table 6$)$ whereas glucose had no effect (Table 3). Our previous experimental observations (Appuhamy et al., 2014) showed acetate and glucose to have no or only a small statistical effect, reducing AMPK phosphorylation by $\sim 8 \pm$ 18 and $23 \pm 10 \%$, respectively. The minimal inhibitory effect of acetate on AMPK phosphorylation estimated herein is reflected in the derived parameter value which had a coefficient of variation of $211 \%$ (Table 6), highlighting the challenge of measuring small responses by immunoblotting and a lack of knowledge of the system.

This final AMPK representation was derived from 55 observations and presented a RMSPE of $33.0 \%$, with

Table 8. Global sensitivity analysis to nutrient and hormone inputs ${ }^{1}$

\begin{tabular}{|c|c|c|c|c|c|}
\hline Item & Evaluation range & Akt & AMPK & mTOR & Casein \\
\hline Insulin & $0.34,10.4$ & 0.97 & 0.00 & 0.02 & 0.01 \\
\hline Acetate & $1.02,3.02$ & & 0.03 & 0.02 & 0.02 \\
\hline Isoleucine & 42,254 & & 0.00 & 0.49 & 0.45 \\
\hline \multicolumn{6}{|c|}{ Sensitivity to model parameters ${ }^{2}$} \\
\hline$K_{\text {Insulin-Akt }}$ & $12.3,18.5$ & 0.03 & 0.0 & 0.0 & 0.0 \\
\hline$k_{\text {AMPK_UP }}$ & $0.49,0.73$ & & 0.63 & 0.0 & 0.0 \\
\hline$J_{\text {AMPK-Acetate }}$ & $1 \times 10^{-5}, 1.5 \times 10^{-5}$ & & 0.02 & 0.0 & 0.0 \\
\hline $\mathrm{mTOR}_{\text {Intercept }}$ & $0.74,1.1$ & & & 0.86 & 0.21 \\
\hline$V_{\mathrm{mTOR}-U P}$ & $0.58,0.88$ & & & 0.06 & 0.02 \\
\hline$K_{\mathrm{Leu}-\mathrm{TOR}}$ & 73,110 & & & 0.0 & 0.00 \\
\hline$K_{\text {Ile-TOR }}$ & 85,127 & & & 0.0 & 0.00 \\
\hline$J_{\text {AMPK-TOR }}$ & $1.02,1.54$ & & & 0.0 & 0.00 \\
\hline
\end{tabular}

${ }^{1} \mathrm{Akt}=$ protein kinase $\mathrm{B} ; \mathrm{mTOR}=$ mammalian target of rapamycin; AMPK = AMP activated protein kinase.

${ }^{2}$ Abbreviations from Table 5 . 
$7.4 \%$ of it arising from slope bias (Table 7). The small effect of acetate on AMPK phosphorylation, despite being its only driver, is reflected in the low sensitivity of phosphorylated AMPK to VFA $(\mathrm{SC}=0.03$, Table 8) and the parameter defining the strength of its inhibitory signal $\left(J_{\text {Acetate-AMPK }}, \mathrm{SC}=0.02\right.$, Table 8$)$. Figure $3 \mathrm{~b}$ illustrates the lack of inhibition of AMPK phosphorylation by acetate from 0 to $5 \mathrm{mmol} / \mathrm{L}$, a range well outside of the normal in vivo range. Instead, AMPK phosphorylation predictions were most sensitive to the mass action rate constant $\left(k_{\text {AMPK-UP }}, \mathrm{SC}=0.62\right.$, Table 8) which, per se, provides no biological insight into its regulation. That is, AMPK phosphorylation would be proportional to the pool size of dephosphorylated AMPK regardless of cellular nutritional and energetic status. Variation in this parameter estimate was relatively small with a confidence interval of 0.52 to 0.68 and a coefficient of variation of $8 \%$ (Table 7). Thus, even though this AMPK submodel presents no mean and minor slope bias, our insight of the relationship between energy substrates, such as acetate or glucose and AMPK, remains limited. A possible explanation for the lack of effect of acetate and glucose on AMPK phosphorylation may be the fact that the data used for parameterization were possibly generated under insufficiently depleted nutritional conditions that led to low AMPK activation levels (i.e., starvation status was not fully induced), as previously noted by the authors (Ap- puhamy et al., 2014). If cellular AA are plentiful, mammary cells could potentially switch to AA oxidation under glucose or acetate deficient conditions, leaving AMPK to be a safety mechanism for extreme general starvation conditions. Appuhamy et al. (2014) found that although both glucose and EAA significantly reduced AMPK phosphorylation in MAC-T cells, only EAA activated mTOR, suggesting a EAA specific effect on mTOR that does not involve AMPK. In addition, variation in EAA concentrations explained $35 \%$ of the changes in mTOR phosphorylation, whereas AMPK activation only explained $16 \%$. Therefore, experimental work is needed to assess the plasticity of mammary cells to shift their metabolism toward EAA oxidation under glucose and acetate shortage, and whether AMPK/ mTOR is the only pathway mediating those effects.

With respect to mTOR, it has been observed experimentally that its phosphorylation (Appuhamy et al., 2011, 2012; Arriola Apelo et al., 2014b) and protein synthesis (Suryawan et al., 2009; Davis et al., 2010) responses are independently regulated by EAA and insulin. In the present work, mTOR phosphorylation data were best described (i.e., lowest AIC) by an M-M function including the additive effects of the BCAA Leu and Ile (Table 4); whereas adding the effect of Akt as a transmitter of insulin stimulus or the effect of AMPK as a signal of energy status did not improve the fit of the model to the mTOR phosphorylation observa-
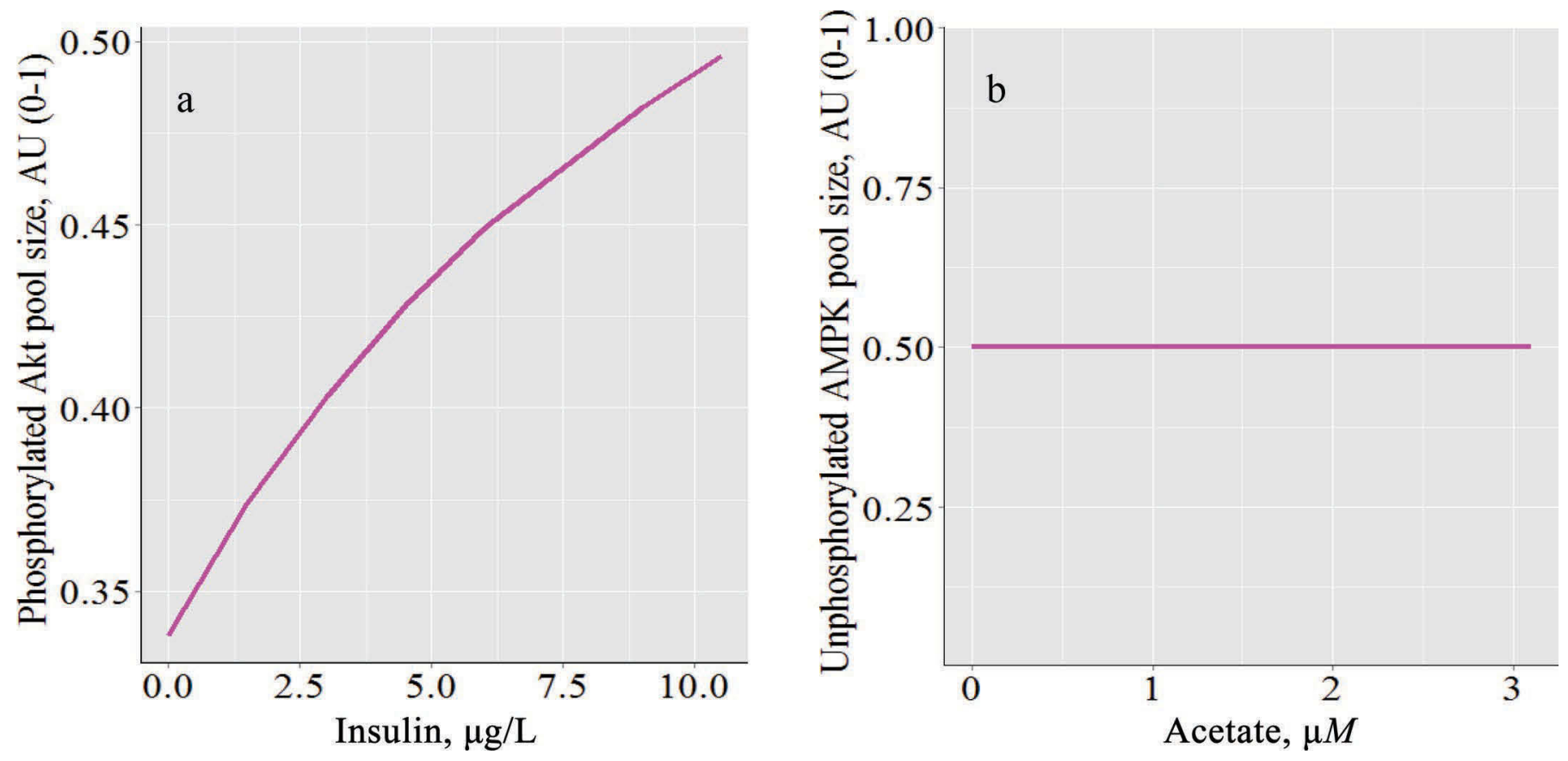

Figure 3. (a) Changes in pool size of phosphorylated protein kinase B [Akt; arbitrary units (AU)] in response to blood insulin concentrations; (b) changes in pool size of unphosphorylated AMP-activated protein kinase (AMPK; AU) in response to blood acetate concentrations. All other model inputs were at reference concentrations. Color version available online. 
tions (Table 4). Our final representation of mTOR had an RMSPE of $28.4 \%$ with no mean or slope bias and was based on 410 observations (Table 7). The affinity constant for Leu and Ile were estimated at $\sim 92$ and 106 $\mu M$ (Table 6), which appears reasonable with respect to mean physiological blood concentrations reported in the literature $(\sim 157 \pm 42$ and $129 \pm 38 \mu \mathrm{M}$, respectively; Hanigan et al., 2002). Figure 4a illustrates the joint effect of AA depicting the predicted increase in mTOR phosphorylation rate $(\mathrm{AU} / \mathrm{h})$ as Leu and Ile concentrations increase from 50 to $600 \mu M$. In this AA concentration range, phosphorylation reaction velocity increases from a basal level (mTOR Intercept $_{\text {t }}$ of 0.9 to 1.4 , which is approximately a $55 \%$ increment. At low Leu concentrations, when Ile is raised from 50 to $600 \mu M$, the model predicts only a small increase from 0.47 to 0.49 in the pool size of phosphorylated mTOR (AU). Similarly, at low Ile concentrations, the marginal effect of Leu is small. But as concentrations of both Ile and Leu increase concomitantly to $600 \mu M$, the compound effect of both AA results in a pool size of 0.55 , as portrayed in Figure $4 \mathrm{~b}$. This is followed by casein synthesis (Figure 4c) where, at low Ile or Leu levels, maximal concentrations of either AA only increase the fractional synthesis rate from 1.75 to $1.87 \% / \mathrm{h}$ (approximately $6.8 \%$ ), but, as both AA increase, casein synthesis rate reaches up to $2 \% / \mathrm{h}$, which is a total increase of $14 \%$. This joint effect Leu and Ile in this representation appears preponderant, as the combined global sensitivity of mTOR phosphorylation to those 2 BCAA was $\sim 0.78$ (combined $\mathrm{SC}=0.49+0.29=0.78$, Table 8). Thus, the cell and MTS data used supported a model in which 2 AA, each one with a small marginal effect in the absence of the other, have a stronger compound effect on protein synthesis when presented simultaneously to the tissue. This observation is contrary to the theory of a single limiting AA and in accordance with previous observations where multiple AA, such as Val, Met, Thr, Arg, or Trp, affected mTOR signaling or casein synthesis to some degree in an additive, simultaneous manner (Appuhamy et al., 2012; Arriola Apelo et al., $2014 b, c)$. Such additivity of milk protein synthetic responses has also been observed when levels of net energy and MP are varied simultaneously (Rius et al., 2010) and reflects the current knowledge at molecular level that, in addition to sufficient supply of EAA to load their respective transfer (t)RNA for peptide elongation during mRNA translation, mTOR and maybe other signaling pathways could stimulate protein synthesis in parallel (El-Haroun et al., 2010). If these tissue-level effects were confirmed experimentally at the animal level, future feeding systems should consider several AA and energy concurrently and integratively rather than considering one single nutrient at a time. However, be- fore we can answer questions from field nutritionists, such as "How much metabolizable leucine or isoleucine should I supplement in addition to my metabolizable lysine and methionine to maximize protein yield?", we must first conduct experimental research at the animal level to confirm and quantify the responses of milk protein yield to simultaneous variation in multiple EAA and compare the quality of those predictions against independent observed data. If such research results in a greater ability to predict reality compared with the limiting AA paradigm embedded in existing feeding systems (e.g., NRC), then the existing biochemical and molecular knowledge, and the mechanistic tissue data would be would provide strong argument and direction for adoption of such a change in field software.

The lack of explanatory power of other EAA, such as Val, Met, Thr, Arg, or Trp with respect to casein synthesis, was inconsistent with the results of our previous experiments. This may be partly explained by the large variability of protein immunoblotting and protein synthesis measures and the fact the synthetic responses to single EAA are rather small as observed by (Arriola Apelo et al., 2014c), where the casein synthesis responses to individual supplementation with Ile, Leu, Thr, or Met increased from about 2.2 to $3.2 \pm 1 \%$. Only when all 4 EAA were considered at the same time did casein synthesis increase dramatically from 1 to $4 \pm$ $1.5 \%$. The latter upper value is $25 \%$ greater than with just 4 EAA, which highlights the apparent role of other EAA in driving casein synthesis despite our inability to derive a model representing all of those EAA.

Figure 5a illustrates the predicted joint effect of the phosphorylated Akt pool size, as a mediator of the insulin stimuli, and Ile extracellular concentrations on mTOR phosphorylation. The axis for phosphorylated Akt spans from 0.35 to 0.55 , corresponding to the phosphorylation levels estimated over the physiological insulin range as displayed in Figure 3a. It is visually apparent that increments in mTOR phosphorylation are far larger with respect to Ile than to phosphorylated Akt. As depicted, at low Ile concentrations the stimuli of insulin is almost null and at high Ile concentrations $(600 \mu M)$ mTOR phosphorylation increases from $\sim 1.25$ to 1.3 (i.e., roughly 4\%), as the phosphorylated Akt pool size goes from 0.35 to 0.55 . This stimuli is much smaller than that of Ile and Leu (Figure 4a) and, at high Ile concentrations, only results in an actual increase from 0.53 to 0.54 or $1.9 \%$ (not shown) in the pool size of phosphorylated mTOR and from 1.95 to $2.0 \% / \mathrm{h}$ in casein synthesis (Figure $5 \mathrm{~b}$ ) when insulin is increased over the assumed physiological range, which contrasts with the much larger effect caused by Ile and Leu. This marginal effect of insulin, as mediated by Akt, reflects the low global sensitivity of mTOR 
phosphorylation to insulin (0.02; Table 8) compared with that of Ile $(\mathrm{SC}=0.49)$ or Leu $(\mathrm{SC}=0.29)$. It is possible that smaller mTOR phosphorylation responses to insulin obtained with MTS presumably due to poor hormone penetration into internal portions of the slices are counteracting the larger responses observed in MAC-T cells (Appuhamy et al., 2011). More work is required with a different cell or animal model to test the derived relationships herein.

Similarly, our data suggests a minimal regulatory effect of AMPK on mTOR compared with Ile and Leu, with the inhibition constant of AMPK ( $J_{\text {AMPK-TOR }}$; i.e., amount of activated AMPK needed to inhibit mTOR activation by $50 \%$, estimated at 1.28 ; Table 6 ). As the phosphorylated AMPK pool size cannot exceed a value of 1 under our model structure (i.e., signaling proteins phosphorylation extent range from 0 to 1 ), the estimated inhibition of mTOR phosphorylation by AMPK is marginal. Because over the assumed physiological blood acetate concentrations the phosphorylated AMPK pool size does not appear to undergo any phosphorylation changes (Figure $3 \mathrm{~b}$ ), this protein seemingly exerts minimal regulatory effects on mTOR phosphorylation rates and pool sizes in that range. This is illustrated in Figure $5 \mathrm{c}$ which shows a steady increase in the mTOR phosphorylation rate with respect to Ile, but no change a.

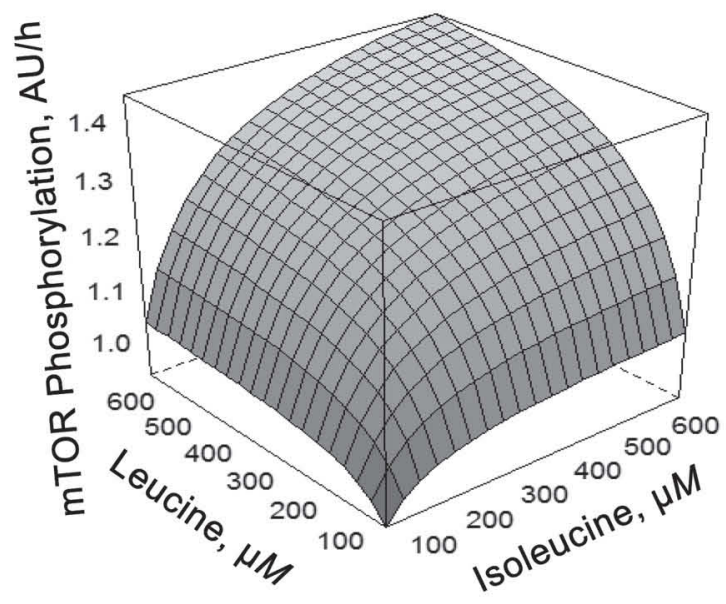

b.

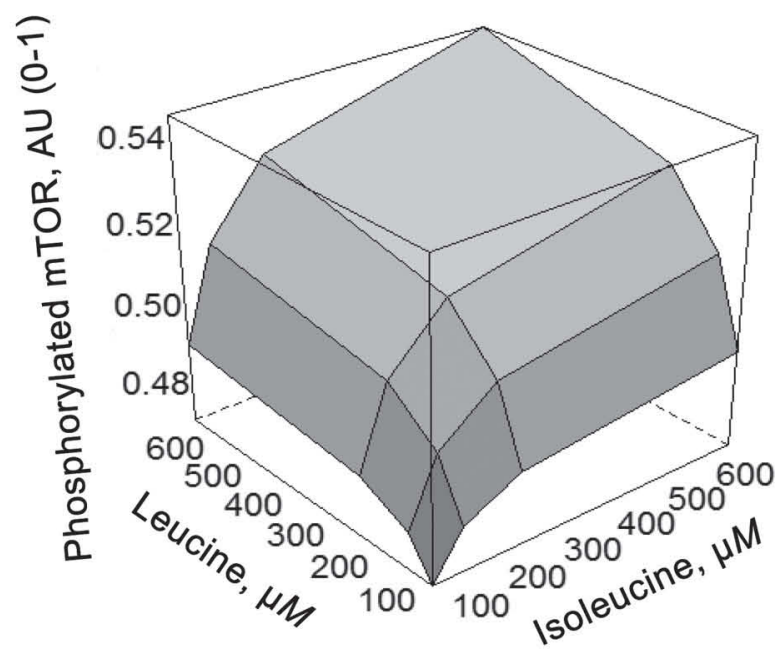

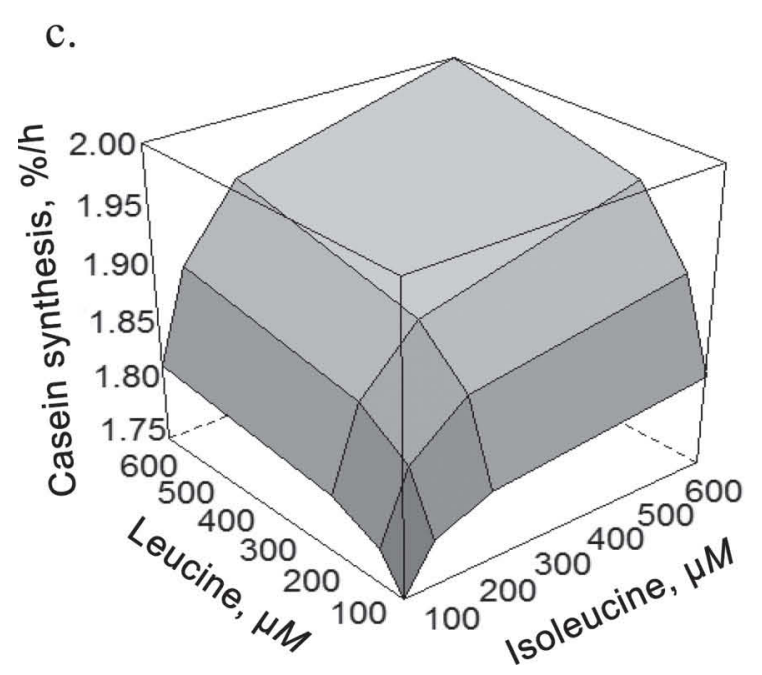

Figure 4. (a) Predicted joint effect of Ile and Leu on mammalian target of rapamycin (mTOR) phosphorylation rate [arbitrary units (AU)/h]. (b) Predicted change in pool size of phosphorylated ion mTOR (AU) at increasing extracellular levels of Ile and Leu. (c) Predicted change in casein synthesis rates at increasing extracellular levels of Ile and Leu. All other model inputs were at reference concentrations. 
a.

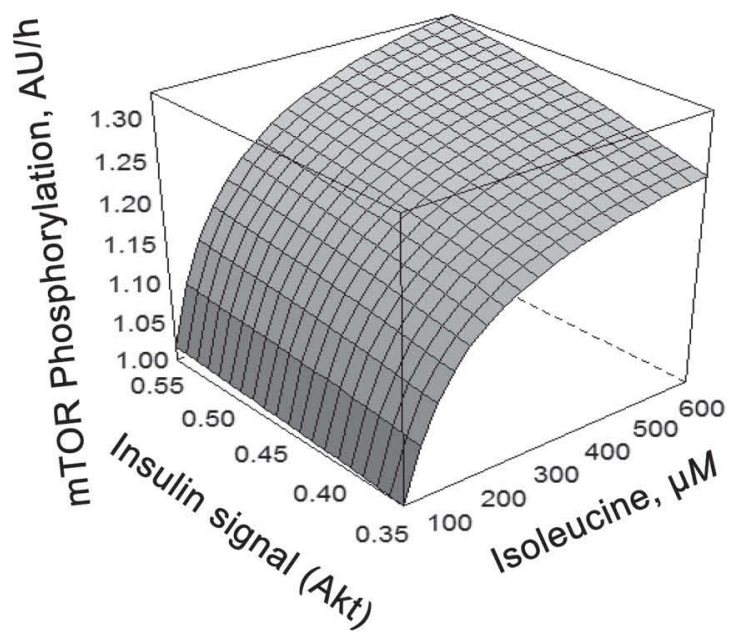

b.

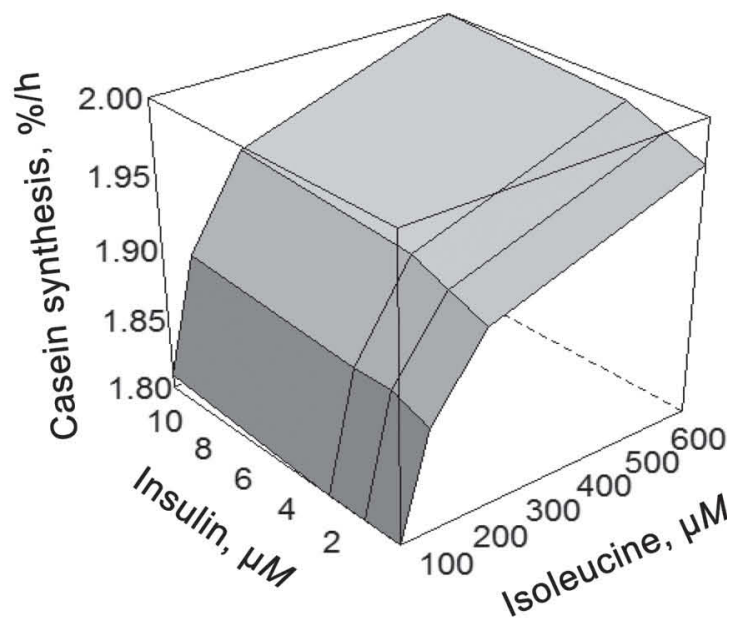

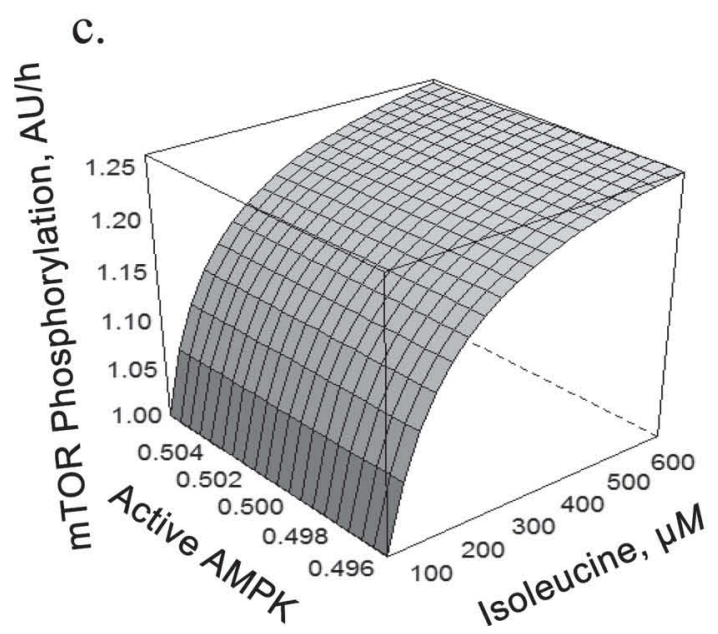

Figure 5. (a) Predicted joint effect of phosphorylated protein kinase B (Akt) and Ile on mammalian target of rapamycin (mTOR) phosphorylation rate [arbitrary units $(\mathrm{AU}) / \mathrm{h}$. (b) Predicted change in casein synthesis rates at increasing extracellular levels of Ile and insulin. (c) Predicted joint effect of phosphorylated AMP-activated protein kinase (AMPK) and Ile on mTOR phosphorylation rate (AU/h). All other inputs were at reference values.

with respect to AMPK in the physiological range of blood acetate concentrations. However, there may be some reinforcement of such a signal through insulin signaling, as one would expect lower energy status in the animal to negatively affect both acetate supply and blood insulin concentrations.

If, however, we consider changes in the phosphorylation mass action rate constant $\left(K_{\mathrm{AMPK}-U P}=0.6\right.$, Table 6 ) to which AMPK is much more sensitive $(\mathrm{SC}=0.62$, Table 8) to examine the relationship between AMPK and mTOR irrespective of acetate, we observe in Figure 6 a that as $K_{\text {AMPK-UP }}$ goes from $\sim 0.1$ to 1 (i.e., 10 -fold) the pool size of unphosphorylated (i.e., active) AMPK decreases by approximately $50 \%$ from 0.8 to under 0.4 . Over this range of unphosphorylated AMPK, the pool size of phosphorylated mTOR rises from 0.53 to 0.55 , which equals a change of $\sim 3.6 \%$ (Figure $6 \mathrm{~b}$ ) and is only approximately $24 \%$ the stimulation caused by Ile and Leu (Figure 4b); whereas, casein synthesis rates rise from 1.94 to $2.02 \% / \mathrm{h}$ (Figure 6c). These changes in mTOR phosphorylation and casein synthesis rates appear rather small given the wide range considered in the AMPK phosphorylation rate constant $K_{\mathrm{AMPK}-U P}$ (10-fold) and in the unphosphorylated AMPK pool 
a.

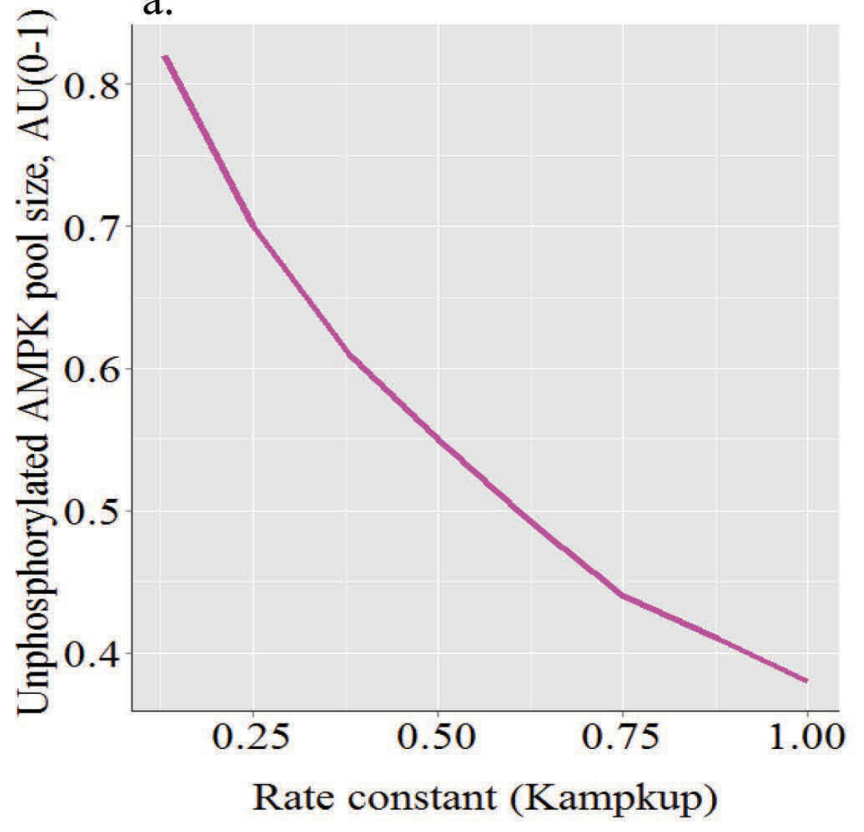

c.

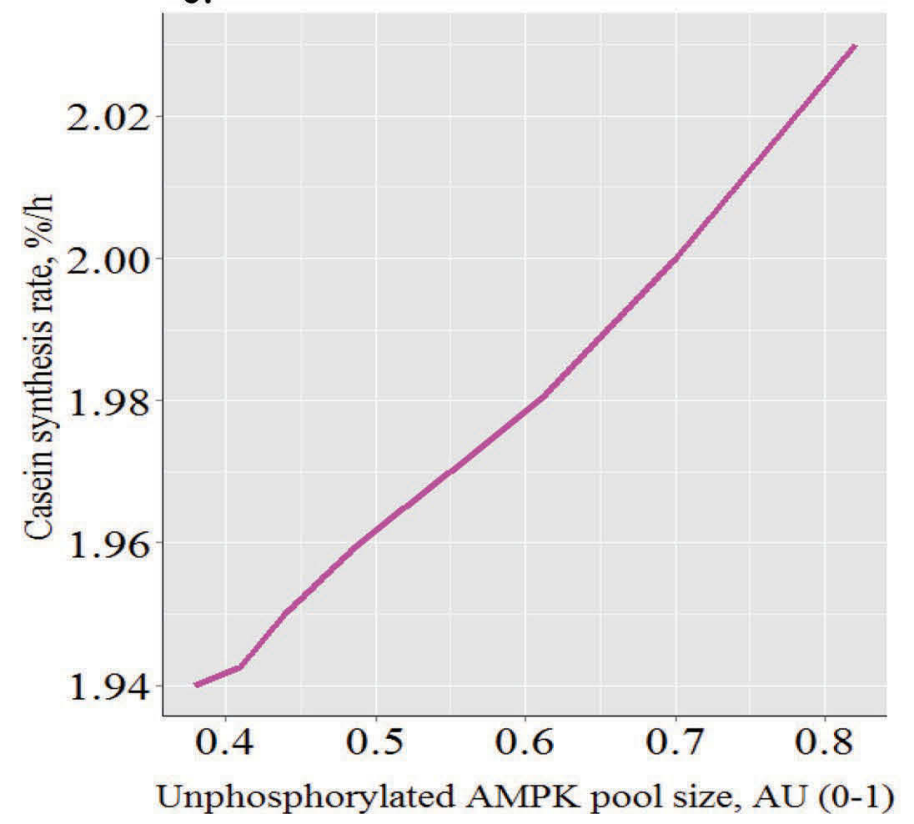

b.

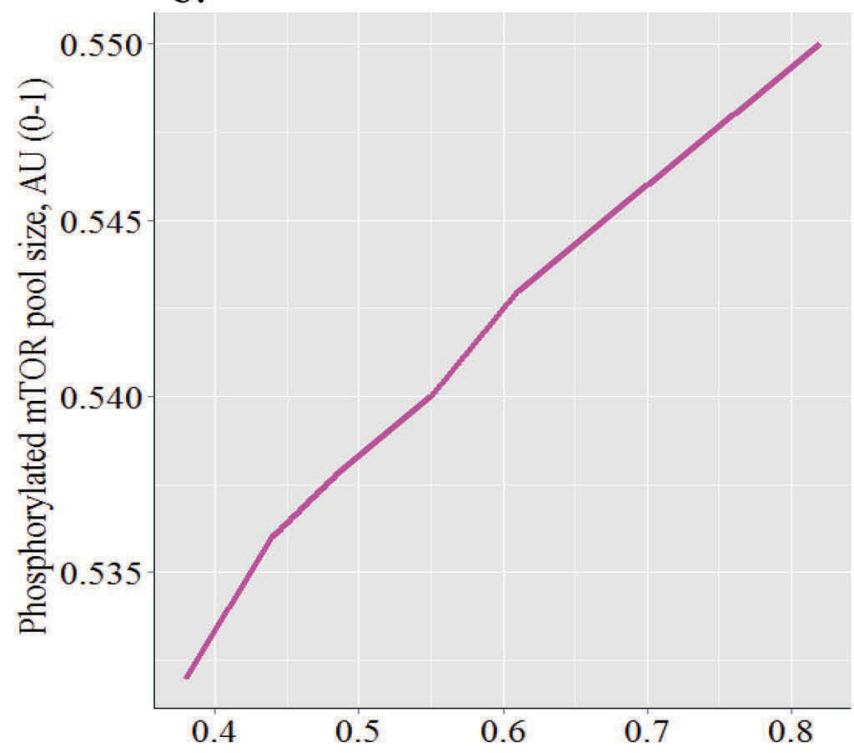

Unphosphorylated AMPK pool size, AU (0-1)

Figure 6. (a) Predicted change in the unphosphorylated AMP-activated protein kinase (AMPK) pool size with respect to the phosphorylation rate constant. (b) Predicted change in the phosphorylated mammalian target of rapamycin (mTOR) pool size with respect to unphosphorylated AMPK. (c) Predicted change in the casein synthesis rate with respect to unphosphorylated AMPK. All of the other inputs were at reference values. $\mathrm{AU}=$ arbitrary units. Color version available online.

size (0.4 to 0.8). Whether that seemingly large degree of variation in $K_{\text {AMPK-UP, }}$, which we have arbitrarily represented, does actually exist in the mammary cells of different cows is unknown; however, the effect of AMPK phosphorylation on protein synthesis through mTOR was marginal compared with EAA, as proposed previously (Appuhamy et al., 2014). Hence, whereas quantitatively defining the dependency of $K_{\mathrm{AMPK}-U P}$ on energy substrates, such as glucose or acetate, and other the factors may be central to quantifying the effect of energy depletion on casein synthesis at the mammary cell level, it is possible that AMPK does not completely 
shut down mTOR phosphorylation in this organ and other pathways support continued mTOR activity (Appuhamy et al., 2014).

The mTOR basal (mTOR Intercept $)$ and maximal phosphorylation $\left(V_{\mathrm{mTOR}-U P}\right)$ rates were estimated at $\sim 0.92$ and 0.73 (Table 6), and mTOR was most sensitive to these 2 parameters with a combined sensitivity coefficient of $0.92\left(\mathrm{mTOR}_{\text {Intercept }} \mathrm{SC}=0.86, V_{\mathrm{mTOR}-U P} \mathrm{SC}=\right.$ 0.06 ; Table 8 ). To the extent that protein synthesis is driven by mTOR phosphorylation, these 2 parameters would set the lower and upper physiological thresholds for the synthetic response in the udder; hence, factors influencing them, such as lactation stage and interactions with cellular nutrient supply, need to be studied to increase our understanding of protein synthesis control by mTOR.

The largest error in the mTOR submodel parameter estimates corresponds to the affinity constants for Akt $\left(K_{\text {Akt-TOR }}\right)$, Ile $\left(K_{\text {Ile-TOR }}\right)$, and AMPK $\left(J_{\text {AMPK-TOR }}\right)$ with confidence intervals of 0.00005 to $0.6,10.4$ to 273 , and 0.29 to 2.55 and coefficients of variation of 85,73 , and $60 \%$, respectively (Table 6). Even though the confidence intervals do not encompass 0 , these estimates are not precise, especially the affinity constant of Akt for mTOR activation. These parameters are central to the definition of mTOR regulation in our model, and consequently, more precise measurements would be very useful to improve those estimates. Nonetheless, a major limitation of current analytical techniques used to measure protein abundance and post-translational state, such as Western immunoblotting, is the lack of precision in those measurements. In our preliminary work, replication of a sample within and across gels generated surprisingly similar variation of close to $30 \%$ both within and across. Obviously, it will be difficult to derive precise parameter estimates given that level of imprecision. Further progress will require either adoption of new, more precise measurement techniques or a massive effort to highly replicate immunoblotting observations to overcome the poor precision of measurement.

Despite the above and based on our data, the effect of Ile and Leu appears to be much larger than that of insulin through Akt and that of acetate through AMPK. More work measuring mTOR phosphorylation in responses to varying energy supply and using perhaps isotopic protein labeling to assess mTOR activity would allow a better definition of the response surface. Downstream of mTOR and separate inclusion of S6k1 or $4 \mathrm{eBP} 1$ during the preliminary analysis (Table 4) appeared to explain casein synthesis rates better than an intercept only model, supporting a role for $\mathrm{S} 6 \mathrm{k} 1$ and $4 \mathrm{eBP} 1$ phosphorylation as integrators of metabolic and hormonal regulation of protein synthesis. However, when added together to the model, the resulting fit of the model to the observed data was inferior to the model containing only mTOR. Because mTOR exerts its regulatory activity through both proteins, we found no justification to preferentially retain only one; therefore, mTOR was included even though it does not act directly. Hence, despite evidence showing that enhanced phosphorylation of S6k1 stimulated by BCAA supplementation is accompanied by downregulation of the translation inhibitory protein 4eBP1(Wang and Proud, 2006), no distinct role for these proteins could be clearly defined within the present representation given the data used. Previous observations in our laboratory have qualitatively supported the hypothesis that EAA supply stimulates translation initiation and elongation by causing phosphorylation of mTOR, S6k1, and 4eBP1 in both bovine mammary epithelial cells and tissue, as well as increased protein synthesis rates in tissue (Appuhamy et al., 2011, Appuhamy et al., 2012) coinciding with observations elsewhere (Davis et al., 2010). Nevertheless, despite the statistical significance observed in those experiments for those relationships, it appears that integrating all effectors and mediators of the mTOR regulatory pathway into a single quantitative representation is a more challenging task to perform due to the seemingly small but additive effects of nutrients, the large multidimensionality of the regulatory process, the correlation among individual proteins within a common pathway, and the variability of the immunoblotting data used to parameterize such equations. It is also certainly possible that the equation forms assessed are not the true ones and further progress could be made if the appropriate equations forms were identified. One key additional point to consider is the type of experimental model, as MAC-T and other bovine mammary cells have been grown, replicated, and shared among many laboratories over several years and are often cultured under extreme supraphysiological supply of nutrients, which could have evolved into quite a different phenotype than that of the intact mammary cell.

With respect to casein synthesis rates, the maximal velocity $\left(V_{\text {CaseinSyn }}\right)$ and the affinity constant of mTOR $\left(K_{\mathrm{mTOR}-\text { Casein }}\right)$ for the casein synthesis reaction were estimated at $53.9 \%$ per hour and 14.1 , respectively. Because phosphorylated mTOR only ranges between 0 and 1 within the model, this affinity constant estimate indicates that synthesis rates do not reach saturation even when mTOR is $100 \%$ phosphorylated and suggests the regulatory affect is in the linear phase of the casein synthesis reaction (Figure 7). In consequence, the pool size of phosphorylated mTOR itself appears 
to be a significant regulator of protein synthesis rates with other factors setting the upper synthetic threshold $\left(V_{\text {CaseinSyn }}\right)$. The estimation errors for both parameters were large according to their coefficient of variation ( $45 \%$ for $V_{\text {CaseinSyn }}$ and $49 \%$ for $k_{\text {mTOR-Casein }}$ ) and confidence interval (35.3-114 for $V_{\text {CaseinSyn }}$ and 8.87-30.7 for $k_{\mathrm{mTOR} \text {-Casein }}$; Table 6 ). Consequently, casein synthesis rates were predicted with the largest error compared with the other variables with RMSPE of $54.9 \%$. Little slope $(3.8 \%)$ and no mean bias supported model adequacy (Table 7 ). The large unexplained variation in casein synthesis could be partly due to the inability of the model to explain the complexity in protein synthesis (i.e., the equation set and their form is too simple); however, based on the information criteria used to evaluate increasingly complex models, addition of Met, Thr, insulin, glucose, or acetate did not explain the casein observations any better. Residual analysis also showed that no other EAA were related to the unexplained variation. But other unknown factors could still play a role. Previously (Appuhamy et al., 2011, 2012, 2014; Arriola Apelo et al., 2014c), we estimated casein synthesis rates under controlled experimental conditions with approximately 20 to $35 \%$ error, which must have been due to unexplained variability and measurement error. We suspect that measurement error is the primary cause of our inability to precisely capture the quantitative effects of the several effectors on mTOR

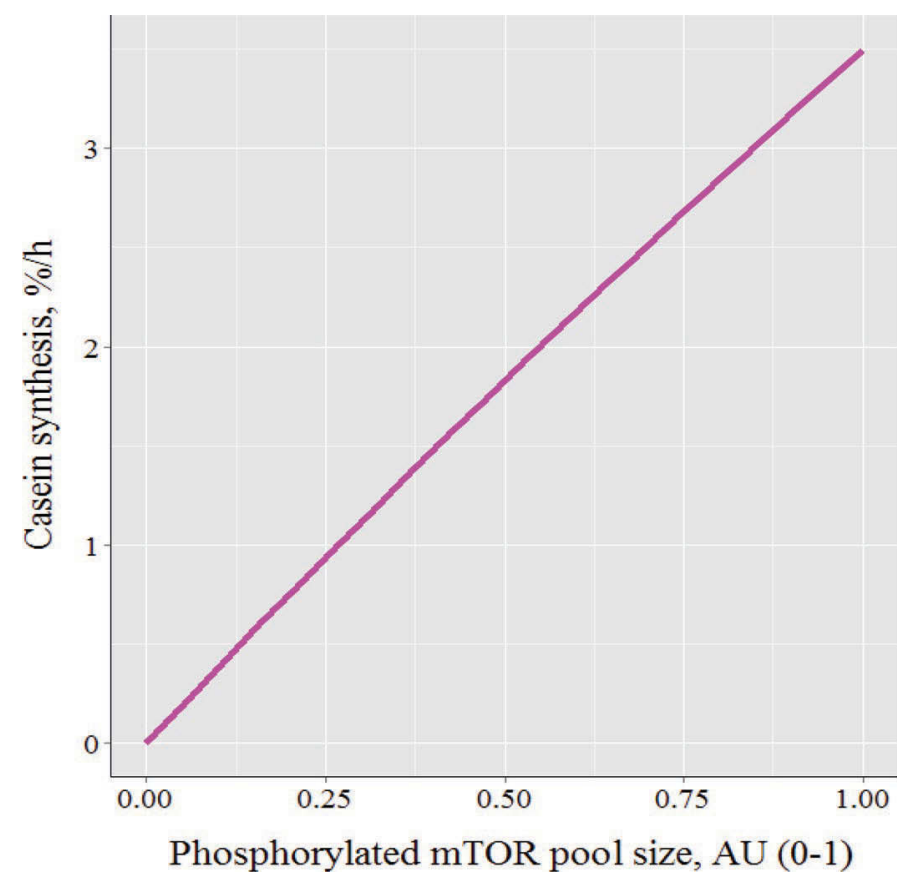

Figure 7. Michaelis-Menten relationship between mammalian target of rapamycin (mTOR) phosphorylation and casein synthesis rate $(\% / \mathrm{h})$. Color version available online. signaling and casein synthesis. Given our efforts to remove inter assay variation, it seems likely at least some of the problem is caused by intraassay variation; but, as noted above, unknown and unrepresented regulatory mechanisms cannot be ruled out.

In spite of this, however, the model appears to reflect well the experimentally known responsiveness of protein synthesis to mTOR signaling, as casein synthesis rates were highly sensitive to Leu $(\mathrm{SC}=0.26)$ and Ile $(\mathrm{SC}=0.50)$, basal mTOR levels $\left(\mathrm{mTOR}_{\text {Intercept }}, \mathrm{SC}=\right.$ $0.21)$, and to the mTOR affinity constant ( $K_{\mathrm{mTOR} \text {-Casein, }}$ $\mathrm{SC}=0.36$; Table 8). This corresponds well with current knowledge that BCAA have a stimulatory effect on translation initiation through mTOR signaling effects on the initial mRNA binding (Kimball and Jefferson, 2006; El-Haroun et al., 2010). On the other hand, casein synthesis rates were not very sensitive to insulin $(\mathrm{SC}=0.01)$ or acetate $(\mathrm{SC}=0.02$, Table 8$)$, mirroring the global sensitivity coefficient profile of mTOR to these signals; integration of experimental cell level data into a model of protein synthesis regulation (ElHaroun et al., 2010), however, has quantitatively shown that insulin at least clearly inhibits $4 \mathrm{eBP}-1$ to stimulate translation initiation, and this effect is additive to stimulation by Leu and EAA sufficiency (i.e., no EAA is limiting). Indeed, insulin has been suggested to mediate an accelerated influx of Leu into muscle cells and the ensuing increase in protein synthesis following EAA consumption (Drummond et al., 2010). Hence, given the consistent response of milk protein output to hyperinsulinemia (Mackle et al., 1999; Bequette et al., 2002), the lack of an insulin effect on mTOR phosphorylation is surprising. However, a hallmark of the in vivo experiments was increased blood flow (Bequette et al., 2002), which is not captured in a cell culture system. Thus the marginal increase in phosphorylated mTOR in response to insulin represented in the model could be magnified by increased substrate supply associated with increased blood flow, including Ile and Leu, to generate a much greater signal and casein synthesis response. In this case, overall milk protein synthesis might be more sensitive to insulin paracrine effects on total EAA flux as affected by blood flow than to the local cellular signaling anabolic effects on transport and synthesis per se. Another important potential aspect of the insulin mediated blood flow increase that would not be captured by this model is the increased transporter activity in the plasma membrane with increased AA delivery (e.g., SLC38A2, SLC7A5). For SLC7A5, a high-affinity transporter of Leu, in particular, upregulation of this transporter during the anabolic phase of the dietary cycle may represent an adaptive response to enhance the mTORC1 growth signal from insulin (Taylor, 2014). But insulin could presumably operate 
through additional transporters as well; because AA uptake in mammary cells occurs through at least 13 different membrane-bound AA transporters with varying degrees of AA promiscuity, this would be a very complex regulatory system to model (Maas et al., 1998). Research is truly needed to understand the local blood flow control of insulin and its coordination with plasma membrane and intracellular signaling events modulating protein synthesis.

The maximal velocity parameter of the casein synthesis reaction $\left(V_{\text {CaseinSyn }}\right)$ appears to have a large influence $(\mathrm{SC}=0.37)$ on protein synthetic rates (Table 8); hence, further study of the biological mechanisms that define the synthetic potential of the udder is justified. Previous work has already indicated that representation of mammary synthetic potential is critical to achieve better predictions and should consider quiescent and active pools of secretory cells, the cycling between these pools, and the effects of nutrition on endocrine signals and enzyme activity of the active cells (Vetharaniam et al., 2003; Hanigan et al., 2008).

\section{CONCLUSIONS}

The model predicted phosphorylated Akt, mTOR, AMPK, and casein synthesis rates with errors of 16.8, $28.4,33.0$, and $54.9 \%$, respectively. In general, predictions were free of bias and most error was due to disturbance error indicating an adequate representation overall. The Akt, mTOR, and casein synthesis submodels provided a good representation of the experimental data. The mTOR was not estimated to be very sensitive to changes in insulin levels, but it was highly sensitive to Ile and Leu, and this signal appears to be successfully transduced by mTOR onto casein synthesis. However, the insulin signal effects may be magnified in vivo as insulin and energy supply to the tissue would generally be positively correlated. The signaling properties and dynamics of AMPK remain to be the subject of further research, even though its potential effect on protein synthesis rates may not be large. In general, better analytical approaches to measure protein abundance and phosphorylation will be necessary to accurately define many of these signaling processes.

\section{ACKNOWLEDGMENTS}

This project was supported in part by Agriculture and Food Research Initiative (AFRI) Competitive Grant no. 2011-03365 from the USDA National Institute of Food and Agriculture (NIFA, Washington, DC), the Virginia Agricultural Experiment Station (Blacksburg, VA), the Hatch Program of the NIFA (NC-2040), USDA. Additional funding was provided by the College of Agriculture and Life Sciences Pratt Endowment at Virginia Tech.

\section{REFERENCES}

Appuhamy, J. A., A. L. Bell, W. A. Nayananjalie, J. Escobar, and M. D. Hanigan. 2011. Essential amino acids regulate both initiation and elongation of mRNA translation independent of insulin in MAC-T cells and bovine mammary tissue slices. J. Nutr. 141:1209-1215.

Appuhamy, J. A., N. A. Knoebel, W. A. Nayananjalie, J. Escobar, and M. D. Hanigan. 2012. Isoleucine and leucine independently regulate mTOR signaling and protein synthesis in MAC-T cells and bovine mammary tissue slices. J. Nutr. 142:484-491.

Appuhamy, J. A., W. A. Nayananjalie, E. M. England, D. E. Gerrard, R. M. Akers, and M. D. Hanigan. 2014. Effects of AMP-activated protein kinase (AMPK) signaling and essential amino acids on mammalian target of rapamycin (mTOR) signaling and protein synthesis rates in mammary cells. J. Dairy Sci. 97:419-429.

Appuhamy, J. A. D. R. N., and M. D. Hanigan. 2011. Modeling the effects of insulin and amino acids on the phosphorylation of mTOR, Akt, and 4EBP1 in mammary cells. Pages 225-233 in Modelling Nutrient Digestion and Utilisation in Farm Animal. D. Sauvant, ed. Wageningen Academic Publishers, Wageningen, the Netherlands.

Arriola Apelo, S. I., J. R. Knapp, and M. D. Hanigan. 2014a. Invited review: Current representation and future trends of predicting amino acid utilization in the lactating dairy cow. J. Dairy Sci. 97:4000-4017.

Arriola Apelo, S. I., L. M. Singer, X. Y. Lin, M. L. McGilliard, N R. St-Pierre, and M. D. Hanigan. 2014b. Isoleucine, leucine, methionine, and threonine effects on mammalian target of rapamycin signaling in mammary tissue. J. Dairy Sci. 97:1047-1056.

Arriola Apelo, S. I., L. M. Singer, W. K. Ray, R. F. Helm, X. Y. Lin, M. L. McGilliard, N. R. St-Pierre, and M. D. Hanigan. 2014c. Casein synthesis is independently and additively related to individual essential amino acid supply. J. Dairy Sci. 97:2998-3005.

Bates, D., and D. Watts. 1988. Non Linear Regression Analysis and Its Applications. Wiley Series In Probability and Mathematical Statistics. John Wiley \& Sons, Hoboken, NJ.

Bequette, B. J., C. E. Kyle, L. A. Crompton, S. E. Anderson, and M. D. Hanigan. 2002. Protein metabolism in lactating goats subjected to the insulin clamp. J. Dairy Sci. 85:1546-1555.

Bibby, J., and H. Toutenburg. 1977. Prediction and Improved Estimation in Linear Models. John Wiley \& Sons, Hoboken, NJ.

Bickerstaffe, R., E. F. Annison, and J. L. Linzell. 1974. The metabolism of glucose, acetate, lipids and amino acids in lactating dairy cows. J. Agric. Sci. 82:71-85.

Blum, J. W., R. B. Wilson, and D. S. Kronfeld. 1973. Plasma insulin concentrations in parturient cows. J. Dairy Sci. 56:459-464.

Boutry, C., S. W. El-Kadi, A. Suryawan, S. M. Wheatley, R. A. Orellana, S. R. Kimball, H. V. Nguyen, and T. A. Davis. 2013. Leucine pulses enhance skeletal muscle protein synthesis during continuous feeding in neonatal pigs. Am. J. Physiol. Endocrinol. Metab. 305:E620-E631.

Burgos, S. A., J. J. Kim, M. Dai, and J. P. Cant. 2013. Energy depletion of bovine mammary epithelial cells activates AMPK and suppresses protein synthesis through inhibition of mTORC1 signaling. Horm. Metab. Res. 45:183-189.

Davis, T. A., A. Suryawan, R. A. Orellana, M. L. Fiorotto, and D. G. Burrin. 2010. Amino acids and insulin are regulators of muscle protein synthesis in neonatal pigs. Animal 4:1790-1796.

Drummond, M. J., E. L. Glynn, C. S. Fry, K. L. Timmerman, E. Volpi, and B. B. Rasmussen. 2010. An increase in essential amino acid availability upregulates amino acid transporter expression in human skeletal muscle. Am. J. Physiol. Endocrinol. Metab. 298:E1011-E1018.

Efron, B., and R. Tibshirani. 1993. An introduction to the bootstrap. Vol. 57. CRC Monographs on Statistics \& Applied Proability. Chapman \& Hall, CRC Press, Boca Raton, FL. 
El-Haroun, E. R., D. P. Bureau, and J. P. Cant. 2010. A mechanistic model of nutritional control of protein synthesis in animal tissues. J. Theor. Biol. 262:361-369.

Fox, J. 2008. Bootstrapping regression models. Pages 587-606 in Applied Regression Analysis and Generalized Linear Models. SAGE Publications, Thousand Oaks, CA.

Hanigan, M. D., L. A. Crompton, B. J. Bequette, J. A. N. Mills, and J. France. 2002. Modelling mammary metabolism in the dairy cow to predict milk constituent yield, with emphasis on amino acid metabolism and milk protein production: Model evaluation. J. Theor. Biol. 217:311-330.

Hanigan, M. D., L. A. Crompton, J. A. Metcalf, and J. France. 2001. Modelling mammary metabolism in the dairy cow to predict milk constituent yield, with emphasis on amino acid metabolism and milk protein production: Model construction. J. Theor. Biol. 213:223-239.

Hanigan, M. D., C. C. Palliser, and A. G. Rius. 2008. Modelling lactation potential in an animal model. Pages 485-506 in Mathematica Modelling in Animal Nutrition. J. France and E. Kebreab, ed. CAB International, Wallingford, UK.

Hanigan, M. D., C. K. Reynolds, D. J. Humphries, B. Lupoli, and J. D. Sutton. 2004. A model of net amino acid absorption and utilization by the portal-drained viscera of the lactating dairy cow. J. Dairy Sci. 87:4247-4268.

Juchem, S. O., F. A. P. Santos, H. Imaizumi, A. V. Pires, and E. C. Barnabé. 2004. Production and blood parameters of Holstein cows treated prepartum with sodium monensin or propylene glycol. J Dairy Sci. 87:680-689.

Kebreab, E., J. France, D. E. Beever, and A. R. Castillo. 2001. Nitrogen pollution by dairy cows and its mitigation by dietary manipulation. Nutr. Cycl. Agroecosyst. 60:275-285.

Kimball, S. R., and L. S. Jefferson. 2006. Signaling pathways and molecular mechanisms through which branched-chain amino acids mediate translational control of protein synthesis. J. Nutr. $136: 227 \mathrm{~S}-231 \mathrm{~S}$.

Lizcano, J. M., and D. R. Alessi. 2002. The insulin signalling pathway. Curr. Biol. 12:R236-R238.

Maas, J. A., J. France, J. Dijkstra, A. Bannink, and B. W. McBride. 1998. Application of a mechanistic model to study competitive inhibition of amino acid uptake by the lactating bovine mammary gland. J. Dairy Sci. 81:1724-1734.
Mackle, T. R., D. A. Dwyer, K. L. Ingvartsen, P. Y. Chouinard, J. M Lynch, D. M. Barbano, and D. E. Bauman. 1999. Effects of insulin and amino acids on milk protein concentration and yield from dairy cows. J. Dairy Sci. 82:1512-1524.

MacRae, J. C., L. A. Bruce, D. S. Brown, and A. G. Calder. 1997. Amino acid use by the gastrointestinal tract of sheep given lucerne forage. Am. J. Physiol. 273:G1200-G1207.

Moshel, Y., R. E. Rhoads, and I. Barash. 2006. Role of amino acids in translational mechanisms governing milk protein synthesis in murine and ruminant mammary epithelial cells. J. Cell. Biochem. 98:685-700.

Press, W. H. 2007. Numerical Recipes: The Art of Scientific Computing. 3rd ed. Cambridge University Press, Cambridge, UK.

R Core Team. 2014. R: A Language and Environment for Statistical Computing. R Foundation for Statistical Computing, Vienna, Austria.

Rius, A. G., M. L. McGilliard, C. A. Umberger, and M. D. Hanigan. 2010. Interactions of energy and predicted metabolizable protein in determining nitrogen efficiency in the lactating dairy cow. J. Dairy Sci. 93:2034-2043.

Saltelli, A., S. Tarantola, and K. P. S. Chan. 1999. A quantitative model-independent method for global sensitivity analysis of model output. Technometrics 41:39-56.

Suryawan, A., P. J. O'Connor, J. Bush, H. Nguyen, and T. Davis. 2009. Differential regulation of protein synthesis by amino acids and insulin in peripheral and visceral tissues of neonatal pigs. Amino Acids 37:97-104.

Taylor, P. M. 2014. Role of amino acid transporters in amino acid sensing. Am. J. Clin. Nutr. 99:223S-230S.

Vetharaniam, I., S. R. Davis, T. K. Soboleva, P. R. Shorten, and G. C. Wake. 2003. Modeling the interaction of milking frequency and nutrition on mammary gland growth and lactation. J. Dairy Sci. 86:1987-1996.

Viollet, B., S. Horman, J. Leclerc, L. Lantier, M. Foretz, M. Billaud, S. Giri, and F. Andreelli. 2010. AMPK inhibition in health and disease. Crit. Rev. Biochem. Mol. Biol. 45:276-295.

Wang, X., and C. G. Proud. 2006. The mTOR pathway in the control of protein synthesis. Physiology (Bethesda) 21:362-369. 